\title{
The small molecule Chicago Sky Blue promotes heart repair following myocardial infarction in mice
}

\author{
Oren Yifa, ${ }^{1}$ Karen Weisinger, ${ }^{1}$ Elad Bassat, ${ }^{1}$ Hanjun Li, ${ }^{2}$ David Kain, ${ }^{1}$ Haim Barr, ${ }^{3}$ Noga Kozer, ${ }^{3}$ \\ Alexander Genzelinakh,, Dana Rajchman, ${ }^{1}$ Tamar Eigler, ${ }^{1}$ Kfir Baruch Umansky, ${ }^{1}$ Daria Lendengolts, ${ }^{1}$ \\ Ori Brener, ${ }^{4}$ Nenad Bursac, ${ }^{2}$ and Eldad Tzahor ${ }^{1}$ \\ 'Department of Molecular Cell Biology, Weizmann Institute of Science, Rehovot, Israel. '2Department of Biomedical \\ Engineering, Duke University, Durham, North Carolina, USA. ${ }^{3} \mathrm{HTS}$ unit, The Nancy and Stephen Grand Israel \\ National Center for Personalized Medicine (G-INCPM), and ${ }^{4}$ Department of Veterinary Resources, Weizmann Institute \\ of Science, Rehovot, Israel.
}

The adult mammalian heart regenerates poorly after injury and, as a result, ischemic heart diseases are among the leading causes of death worldwide. The recovery of the injured heart is dependent on orchestrated repair processes including inflammation, fibrosis, cardiomyocyte survival, proliferation, and contraction properties that could be modulated in patients. In this work we designed an automated high-throughput screening system for small molecules that induce cardiomyocyte proliferation in vitro and identified the small molecule Chicago Sky Blue 6B (CSB). Following induced myocardial infarction, CSB treatment reduced scar size and improved heart function of adult mice. Mechanistically, we show that although initially identified using in vitro screening for cardiomyocyte proliferation, in the adult mouse CSB promotes heart repair through (i) inhibition of CaMKII signaling, which improves cardiomyocyte contractility; and (ii) inhibition of neutrophil and macrophage activation, which attenuates the acute inflammatory response, thereby contributing to reduced scarring. In summary, we identified CSB as a potential therapeutic agent that enhances cardiac repair and function by suppressing postinjury detrimental processes, with no evidence for cardiomyocyte renewal.

Conflict of interest: The authors have declared that no conflict of interest exists.

Copyright: (c) 2019, American Society for Clinical Investigation.

Submitted: February 7, 2019

Accepted: October 3, 2019

Published: November 14, 2019

Reference information: /CI Insight. 2019;4(22):e128025.

https://doi.org/10.1172/jci.

insight.128025.

\section{Introduction}

The heart is the first organ to develop in the embryo and from then on, it does not stop contracting and pumping, ensuring blood circulation throughout the body. For the heart to function, the cardiac cells themselves require constant blood supply delivered via a network of coronary vessels. Blockage of these vessels often leads to a massive ischemic event in the cardiac tissue known as myocardial infarction (MI). In addition to extensive cardiomyocyte loss, such injuries result in impaired cardiomyocyte contraction, formation of a fibrotic scar, cardiac hypertrophy, and remodeling. In the years following the first MI incident, patients are prone to recurrent MI events and gradual progression toward heart failure $(1,2)$. A major cause of this unfortunate scenario is the fact that the adult mammalian heart regenerates very poorly after injury — what places ischemic heart diseases as the leading cause of death worldwide (2).

Over the years, extensive research and resources have been invested in elucidating strategies for heart recovery after injury aiming at regenerating the cardiac muscle tissue $(3,4)$. One of these strategies involves transplantation of several cell types including skeletal muscle myoblasts (5), various types of stem cells (6, $7)$, and induced cardiomyocytes $(8,9)$. In general, these studies showed moderate improvement of heart function but poor survival and integration of the cells injected into the myocardium. The beneficial effects of cardiac cell therapy have not been proven clinically (10).

Another strategy for heart regeneration is the induction of endogenous cardiomyocyte proliferation. This approach is based on the fact that in cases where cardiac regeneration naturally occurs, like the neonatal mouse $(11,12)$, zebrafish $(13,14)$, or newt $(15,16)$, it involves proliferation of healthy mononucleated cardiomyocytes that repopulate the injured zone. In mice, transition of mononucleated to bi- or multinucleated cardiomyocytes occurs during the first week after birth, but a modest population of mononucleated 
cardiomyocytes persists in the adult mouse heart as well $(17,18)$. Several studies showed signs of adult cardiomyocyte proliferation induced by manipulation of cell cycle genes (19-22), intracellular signaling (23-27), or the extracellular environment $(28,29)$; however, current methods to show bona fide cardiomyocyte division are not conclusive $(30,31)$. Another strategy to restore cardiac tissue is by induction of fibroblast-to-cardiomyocyte transdifferentiation by transfecting cardiac fibroblasts with transcription factors that drive cardiac development (32). Overall, the ability of the mentioned approaches to regenerate the heart and repair its function in humans requires clinical validation.

A different approach for heart recovery following injury is to overcome secondary injuries that follow MI. Modulation of the inflammation and immune response can be linked to the severity of fibrosis and other pro-regenerative effects (reviewed in refs. 33 and 34). Yet, the exact molecular crosstalk between immune and cardiac cells is far from clear. Other studies aiming to reduce secondary injuries of the heart attempted to restore the efficient contraction of the myocardium, which is decreased after MI $(35,36)$.

The studies described above have made profound contributions to our understanding of cardiac repair processes in mammals, albeit not in humans. Unfortunately, there is no safe and efficient treatment for patients suffering from heart failure $(1,2)$.

In this study we aimed to identify small molecules that could serve as novel cardiac repair agents after MI. For this, we designed a high-throughput screening system for small molecules that induce postnatal day 8 (P8) cardiomyocyte proliferation in vitro and found the small molecule Chicago Sky Blue 6B (CSB). We next demonstrated that CSB treatment reduces scar size and improves cardiac function of adult mice after MI, but with no evidence for the induction of cardiomyocyte proliferation in vivo. In adult mice, CSB improves cardiac function by 2 different mechanisms: (a) inhibition of CaMKII, which improves cardiomyocyte contraction; and (b) inhibition of neutrophil and macrophage activation, which could explain the reduction in scar size. We suggest that CSB could be considered as a novel cardiac repair agent that improves heart function by modulating the immune response and improving cardiomyocyte contractility.

\section{Results}

Small-molecule screen identifies CSB as a molecule that induces P8 cardiomyocyte proliferation. To identify small molecules that can stimulate postmitotic cardiomyocyte proliferation and cardiac repair we developed an in vitro high-throughput screening system that measures cardiomyocyte number in live cardiac cell culture. To evaluate true cardiomyocyte proliferation and avoid false-positive results that rise from karyokinesis in the absence of cytokinesis we designed a system that counts the actual number of cardiomyocytes and does not rely on expression of proliferation markers. The system facilitated rapid and unbiased testing of approximately 10,000 compounds. To perform this screen, we crossed myosin heavy polypeptide 6-Cre (Myh6Cre) mice with Rosa26-tdTomato mice to generate MHC:Tomato mice, resulting in cardiomyocyte-specific expression of the Tomato reporter protein. Although mouse cardiomyocytes are able to proliferate during the first week after birth, P8 cardiomyocytes were shown to exit the cell cycle (11). We isolated cardiac cells from P8 MHC:Tomato mice and plated them in 384-well plates. One day later we added 1 tested compound to each well (day 0). The number of cardiomyocytes in each well was counted daily for 6 days after compound administration using automated fluorescence microscopy and the change in cardiomyocyte number in each well was plotted (Figure 1A). Using this screen, we identified several compounds that increased cardiomyocyte number (Table 1).

To validate the positive hits, we performed further in vitro experiments with the most effective compounds and identified CSB, a small molecule $(992.8 \mathrm{~g} / \mathrm{mol}$; for structure see Figure $1 \mathrm{~F})$ that repeatedly induced P8 cardiomyocyte division in culture. CSB is an azo-dye used as a counterstain for reducing background in immunofluorescence staining. CSB was shown to interact with several proteins, including the vesicular glutamate transporter (VGLUT) (37), macrophage migration inhibitory factor (MIF) (38), RAD1 (39), and ubiquitin (40). To validate the proliferative effect observed in the screen we first repeated the screen assay several times. We found that 6 days of incubation with CSB resulted in a $4.6 \%$ increase (1.046-fold change) in the number of cardiomyocytes, while the cardiomyocyte number in the untreated cultures remained constant (Figure 1B). Next, we incubated P8 cardiac cells with CSB for 4 days in vitro and stained for the cell cycle reentry marker Ki67 (41). While in the untreated cultures $0.39 \%$ of the cardiomyocytes were $\mathrm{Ki}^{+} 7^{+}$, CSB treatment increased the number of $\mathrm{Ki}^{+} 7^{+}$cardiomyocytes to $0.89 \%$ (Figure 1C).

The decline in the proliferative capacity of cardiomyocytes during the first week after birth is associated with the appearance of binucleated cardiomyocytes, which are considered to be terminally differentiated 
A

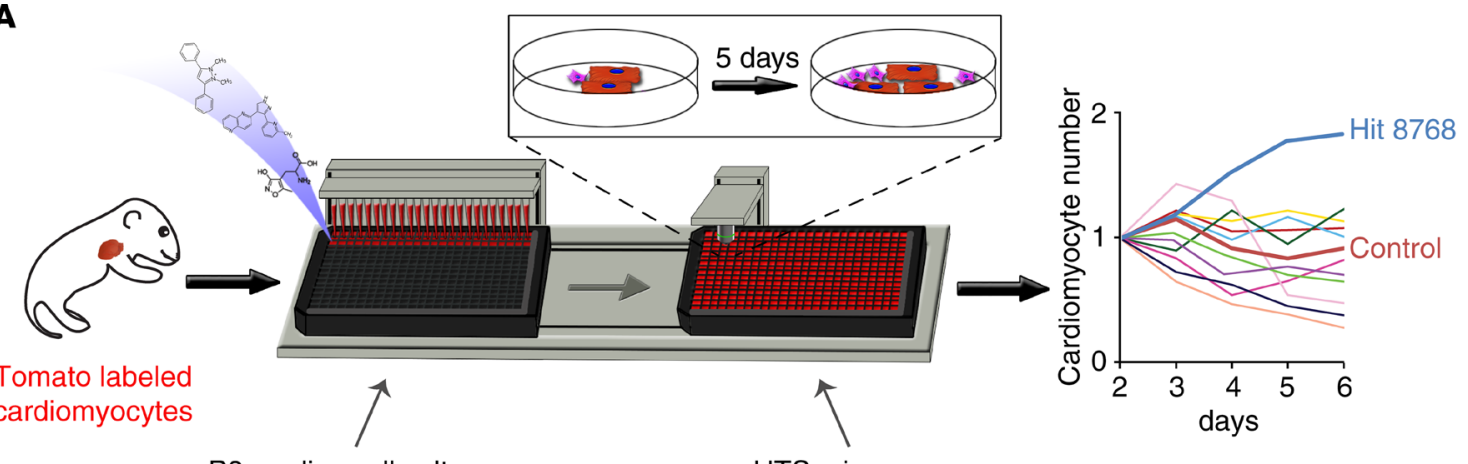

P8 cardiac cell culture

HTS microscopy

Small molecule administration

Automated cardiomyocyte counting

B Number of cardiomyocytes

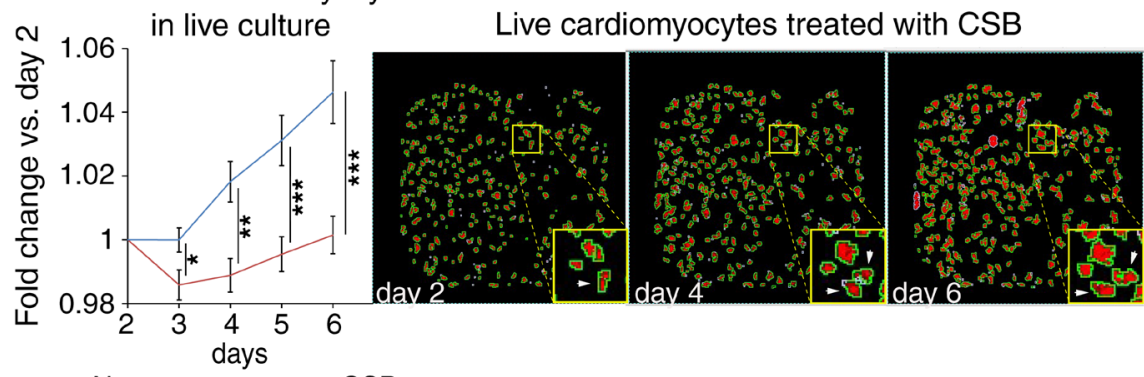

- No treatment - CSB

C

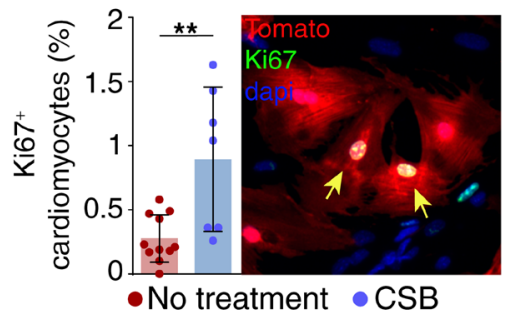

F Number of cardiomyocytes

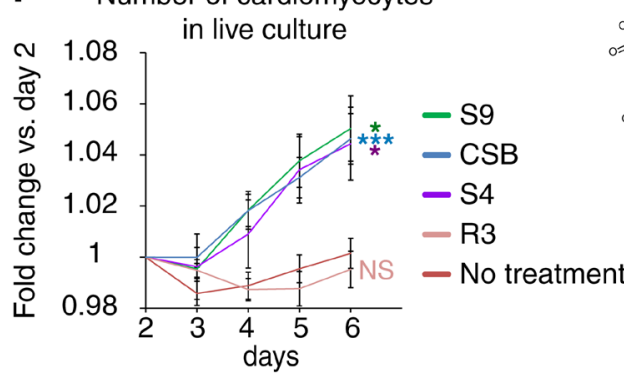

D

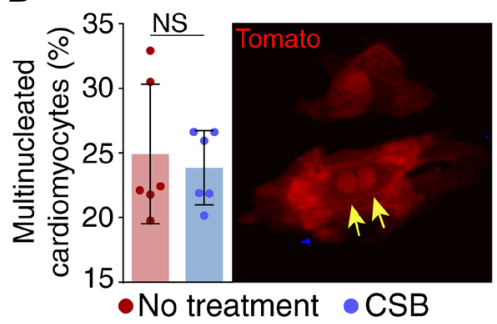

E

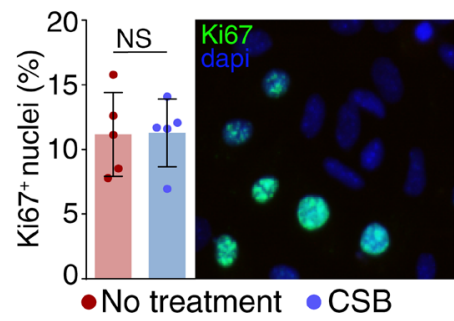

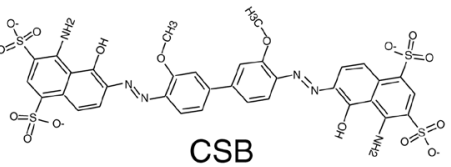

CSB

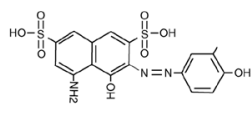

S9

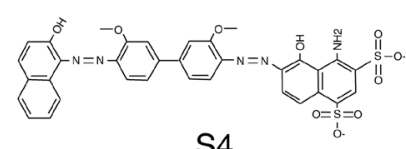

S4

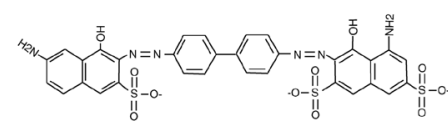

R3

Figure 1. Small-molecule screen identifies Chicago Sky Blue 6B as a molecule that induces cardiomyocyte proliferation. (A) Schematic representation of the high-throughput screening system. P8 cardiac cells were plated in 384-well plates and introduced to different small molecules. Using automated high-throughput microscopy, the cardiomyocytes in each well were counted daily and compared to the number of cardiomyocytes in the same well in the beginning of the experiment. (B) Repeated measurements for validation of the screen results. Number of P8 cardiomyocytes in live culture relative to day 2 (no treatment, $n=20$; Chicago Sky Blue 6B [CSB], $n=16$; data are presented as mean \pm SEM, unpaired 2-tailed Student's $t$ test). (C) Percentage of Ki67 ${ }^{+}$P8 cardiomyocytes normalized to total cardiomyocytes after 4-day incubation with CSB (no treatment, $n=11$; CSB, $n=7$; mean \pm SD, unpaired 2-tailed Student's $t$ test). (D) Percentage of multinucleated P8 cardiomyocytes normalized to total cardiomyocytes after 4-day incubation with CSB ( $n=6$ for each group, mean \pm SD, paired 2-tailed Student's $t$ test). (E) Percentage of Ki67+ nuclei normalized to total nuclei in P8 cardiac cells after 4-day incubation with CSB ( $n=5$ for each group, mean \pm SD, paired 2-tailed Student's $t$ test). (F) Number of P8 cardiomyocytes in live culture relative to day 2. Cells were treated with compounds that share structural similarity with CSB (no treatment, $n=20$; CSB, $n=16$; S9, $n=4$; S4, $n=6$; R3, $n=3$; mean \pm SEM, 1-way ANOVA and Dunnett's post hoc test). The colored asterisks represent the significance of the difference for each compound from the nontreated culture. The data for no treatment and CSB-treated cultures in panels $\mathbf{B}$ and $\mathbf{F}$ are an average of the same samples. For all panels: ${ }^{*} P<0.05,{ }^{* *} P<0.01,{ }^{* *} P<0.001$. NS, not significant. 
Table 1. The 5 leading hits discovered by the screen

\begin{tabular}{lccccc}
\hline & \multicolumn{2}{c}{ Positive Hits } & & \\
Compound & Brompheniramine & Jasminum $^{A}$ & ATPA & Trimipramine & Chicago Sky Blue 6B (CSB) \\
CAS number & $980-71-2$ & & $140158-50-5$ & $138283-61-1$ & $2610-05-1$
\end{tabular}

AJasminum was supplied by AnalytiCon Discovery and has no published CAS number.

(17). We tested whether the increase in $\mathrm{Ki}^{+} 7^{+}$cardiomyocytes induced by CSB treatment indicates cardiomyocyte proliferation and not an increase in cardiomyocyte binucleation. We found that 4-day incubation of P8 cardiomyocytes with CSB did not affect the percentage of binucleated cardiomyocytes, normalized to the total cardiomyocytes (Figure 1D), further supporting the notion that the increase in $\mathrm{Ki}^{-} 7^{+}$cardiomyocytes indicates true proliferation.

After 5 days in culture, the majority of the cells are not cardiomyocytes but rather cardiac fibroblasts, endothelial cells, and other non-cardiomyocytes. To evaluate whether the proliferative effect of CSB is specific to cardiomyocytes we measured the number of total nuclei that are Ki67 $7^{+}$. CSB treatment did not change the percentage of $\mathrm{Ki} 67^{+}$nuclei relative to total nuclei number (the number of $\mathrm{Ki} 67^{+}$cardiomyocytes is negligible in comparison to the rest of the cells) (Figure 1E), suggesting a specific effect of CSB on cardiomyocytes. To further confirm CSB activity we performed a structure-activity relationship (SAR) test. We examined the ability of 3 molecules that share structural similarity with CSB to induce cardiomyocyte division using the same screen. Of the 3 compounds, S9 and S4 increased cardiomyocyte number similarly to $\mathrm{CSB}$, whereas the molecule $\mathrm{R} 3$ bears a small structural change that hinders its ability to induce cardiomyocyte proliferation (Figure 1F). These results suggest that a specific structure in the backbone of CSB is necessary to induce cardiomyocyte proliferation. In summary, using a high-throughput screen we identified the small molecule CSB that promotes proliferation of P8 cardiomyocytes in vitro.

CSB reduces scar size and improves cardiac function of adult mice after MI. Next, we tested the effect of CSB on cardiac function in vivo in adult mice. For this, we induced MI in 12-week-old mice by performing left anterior descending artery (LAD) ligation and injected the mice with CSB or PBS as a control. The first injection was intramuscular (i.m.) directly into the myocardium immediately after the MI (Supplemental Video 1; supplemental material available online with this article; https://doi.org/10.1172/jci.insight.128025DS1), followed by 3 additional intravenous (i.v.) injections into the tail vein 2, 4, and 6 days after injury. Eight days after MI we fixed, sectioned, and stained the hearts with Masson's trichrome, a collagen stain, as a proxy for the fibrotic scar (Figure 2, A and B). Injured mice in both groups had fibrotic tissue at the area of the injury. In the PBS-treated mice the fibrosis expanded toward the apex, the left ventricle was dilated, and the ventricle wall was very thin; however, in the CSB-treated mice the scar was more restricted to the area of the injury (Figure 2B). Measurements of the infarcted zone showed that CSB treatment reduced infarcted area in the hearts of adult mice 8 days after injury (Figure 2C). Classification of the mice into 3 groups by severity of the infarction revealed that while PBS-treated mice displayed varying severities, 8 out of the 9 CSB-treated mice had only mild infarction with fibrosis localized only to the ligation site (Figure 2D and Table 2).

To test the effect of CSB over time, we performed a similar experiment and analyzed the hearts 21 days after MI (Figure 2, A and E). The results showed a wide range of infarction severities following both treatments. This high variation led to an insignificant change in the average infarction size between the 2 treatment groups (Figure 2F). Nevertheless, in the CSB-treated group there were more mice with mild infarction (68.75\%) and fewer mice with severe infarction (18.75\%) relative to PBS-treated controls $(50 \%$ and $30 \%$, respectively) (Figure $2 \mathrm{G}$ and Table 2). Taken together, our results suggest that CSB treatment reduces cardiac scar size in adult mice following MI.

In addition to quantification of the scar size, we used echocardiography to evaluate cardiac function of treated and control mice following injury. Two days after MI the cardiac ejection fraction (EF) and fractional shortening (FS) were similar in CSB- and PBS-treated mice. However, 4 days after MI, EF and FS values declined sharply in PBS-treated mice, while CSB-treated mice were protected from this deterioration (Figure 3, B and C). The difference in heart function persisted until the end of the experiment 21 days after MI. At that point, the EF and FS of CSB-treated mice were closer to those of uninjured mice than to those of PBS-treated mice (Figure 3, B and C). We validated these results using a different echocardiography 

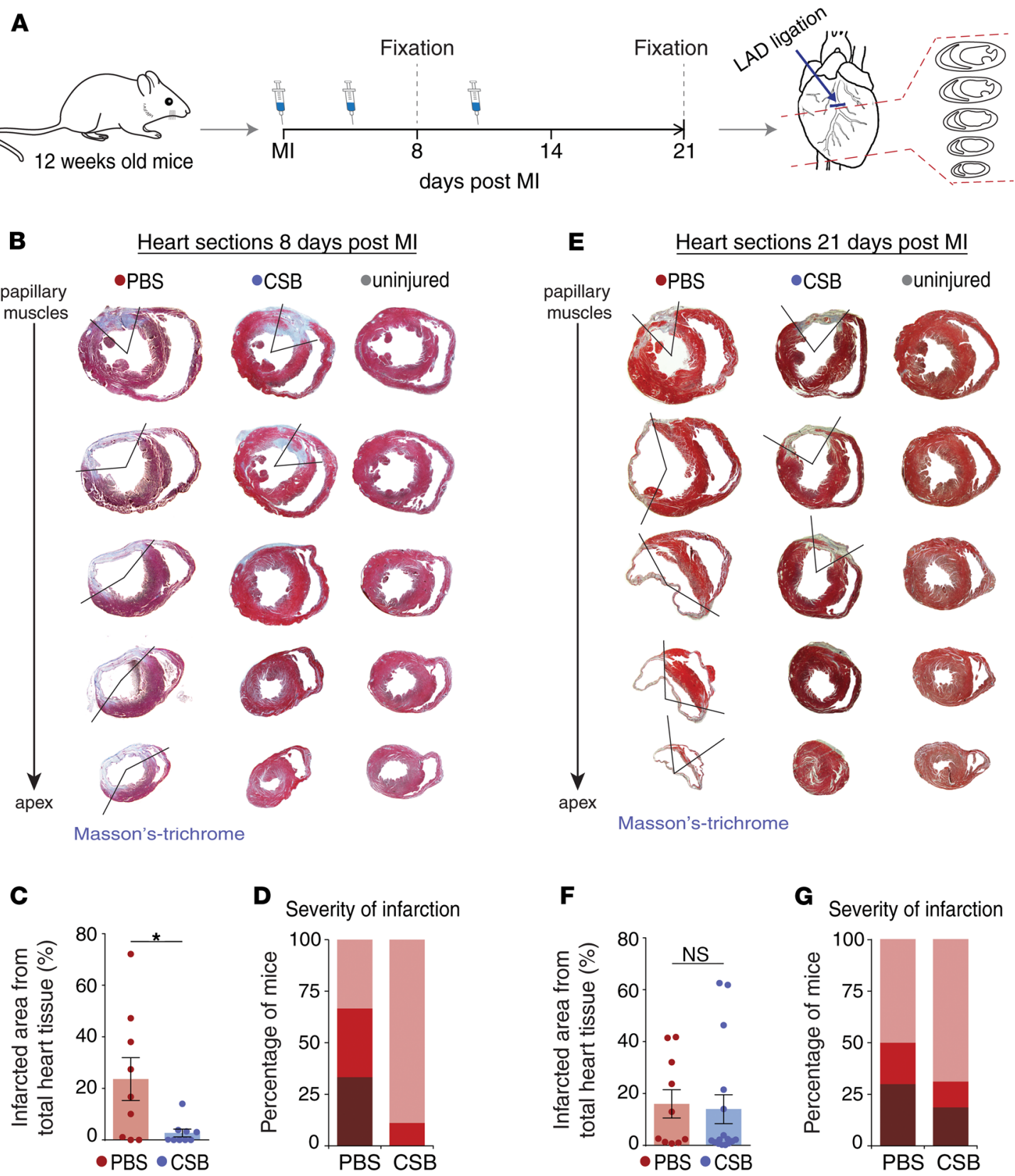

- Mild (infarcted area < 10\%) - Moderate (infarcted area is 10\%-30\%) - Severe (infarcted area > 30\%)

Figure 2. CSB reduces scar size in adult mouse heart following MI. (A) Schematic representation of the MI experiment timeline. Twelve-week-old mice underwent $\mathrm{MI}$ and were injected with PBS or CSB. The first injection was given directly into the myocardium immediately after LAD ligation. Additional injections were given i.v. into the tail vein during 2 weeks after the injury - 3 injections/week. The hearts were fixed 8 or 21 days after MI, sectioned, and stained with Masson's trichrome. (B-D) Quantification of the infarcted area in adult mouse hearts 8 days after MI. (B) Representative images of the infarcted hearts. (C) Quantification of the infarcted area as percentage of total heart tissue (data are presented as mean \pm SEM, 2-tailed Student's $t$ test). (D) Heart classification by the severity of infarction. For $\mathbf{C}$ and $\mathbf{D}: n=9$ for each group. (E-G) Quantification of the infarcted area in adult mouse hearts 21 days after MI. (E) Representative images of the infarcted hearts. The PBS-treated heart has severe infarction, whereas the CSB-treated heart has moderate infarction. (F) Quantification of the infarcted area as percentage of total heart tissue (mean \pm SEM, 2-tailed Student's $t$ test). (G) Heart classification by the severity of infarction. For $\mathbf{F}$ and $\mathbf{G}$ : PBS, $n=10$; CSB, $n=16$. For all panels: ${ }^{*} P<0.05$. NS, not significant.

system (Supplemental Figure 1, A and B). Previous studies showed that CSB has several biological effects (37, 42-46). Therefore, we examined the mice for adverse side effects at the end of the in vivo experiment, 21 days after MI. Due to the similarity of skeletal muscle to the cardiac tissue and previous studies showing that CSB affects the nervous system, the examination focused mainly on lesions and tumors in skeletal muscles and brain. Mice that were treated with CSB did not exhibit any pathological differences compared 
Table 2. Mice classification by the severity of infarction

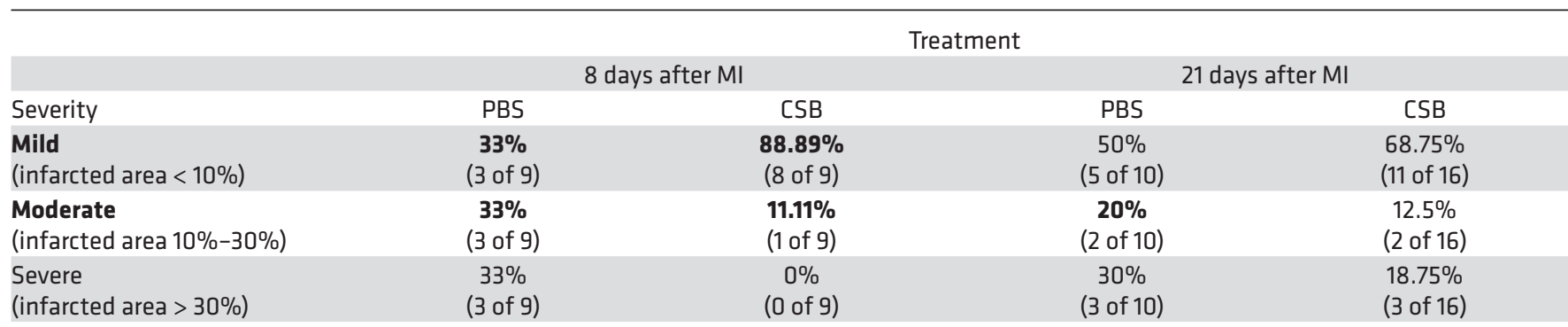

to the PBS-treated mice (data not shown).

Finally, we evaluated the level of cardiomyocyte proliferation in vivo in the injured mice. We induced MI in adult mice and injected CSB or PBS as described above. We fixed the hearts 4 and 8 days after MI and stained for Ki67. Surprisingly, CSB treatment did not increase the number of $\mathrm{Ki}^{+} 7^{+}$cardiomyocytes in vivo after MI (Figure 3D and Supplemental Figure 1C). This result raises the question of how CSB reduces scar size and improves heart function of adult mice without inducing cardiomyocyte proliferation.

CSB inhibits CaMKII activation and improves cardiomyocyte contraction. CaMKII is an intracellular signaling protein that has been intensively investigated as a target for cardiac therapeutic treatment $(47,48)$. CaMKII protein levels and activity rise in injured hearts in correlation with adverse cardiac remodeling ( 35 , 36, 49). Accordingly, CaMKII inhibition was shown to reduce fibrosis (50), prevent cardiac hypertrophy, improve cardiomyocyte contraction, and restore heart function following MI $(35,36,51)$. To elucidate CSB's mechanism of action, we isolated cardiac cells from P8 mice, cultured them for 4 days with CSB, and lysed the cells for Western blot analysis for CaMKII. We found that CSB modestly decreased the level of total CaMKII protein and, to a larger extent, the level of the active phospho-CaMKII (Figure 4A). We next examined whether CaMKII inhibition mimics CSB's effects in cultured P8 cardiac cells by treating them with KN62 - a CaMKII-specific inhibitor. Similarly to CSB, KN62 administration increased cardiomyocyte number in a dose-dependent manner (Figure 4B). Next, we tested whether CSB inhibits CaMKII in vivo in the hearts of adult mice following MI. Five days after MI, CSB blocked the increase of phospho-CaMKII without changing the levels of the total CaMKII protein (Figure 4C).

To determine whether CaMKII inhibition can exert a positive effect on heart function after MI, we induced $\mathrm{MI}$ in adult mice, injected them with $\mathrm{KN} 62$, and recorded heart function at 2, 4, and 21 days after MI. Consistent with CSB treatment, KN62-treated mice had higher EF compared with PBS-treated controls, closer to uninjured mice than to the PBS-treated group (Figure 4D).

To test whether phospho-CaMKII inhibition occurred in cardiomyocytes or non-cardiomyocytes we isolated cardiac cells from adult mice and plated them in vitro for 2 passages, after which there were no cardiomyocytes in the culture and most of the cells were cardiac fibroblasts. We incubated the cells for 4 days with CSB and blotted the lysates for total CaMKII and phospho-CaMKII levels. CSB did not change the levels of total CaMKII or phospho-CaMKII in non-cardiomyocytes, suggesting that the inhibition that was observed in adult mouse hearts results from lower levels of phospho-CaMKII in cardiomyocytes (Supplemental Figure 2).

Upregulation of CaMKII after MI was shown to disrupt intracellular calcium handling in cardiomyocytes (52). This leads to inefficient contraction of cardiomyocytes, with weaker and slower twitching (35, 53). To test CSB's effect on intracellular calcium handling and cardiomyocyte contractility we utilized cardiobundles - functional 3D engineered cardiac tissue made from neonatal rat ventricular myocytes (NRVMs). Within 14 days of culture, the cardiobundles acquire near-adult functional output and structural and molecular characteristics, including cell density, cardiomyocyte alignment, and gene and protein expression (54). CSB was added daily to the cardiobundle medium from day 9 to 14, at which point we examined the calcium transient and contractile force generation. CSB treatment of NRVM cardiobundles reduced the protein levels of total CaMKII and phospho-CaMKII, similar to the changes observed in P8 mouse cardiac cell culture and adult mouse heart after CSB treatment (Figure 5A). To examine calcium transient dynamics, we transduced cardiobundles with a lentivirus driving the cardiac-specific expression of a calcium sensor, GCaMP6 (55-57), or treated them with Fluo-4 dye followed by electrical pacing and imaging. GCaMP6 and Fluo-4 dye both emit fluorescent light when bound to free calcium in the cytoplasm. Cardiobundles treated with CSB exhibited larger calcium transient amplitude (measured 
A
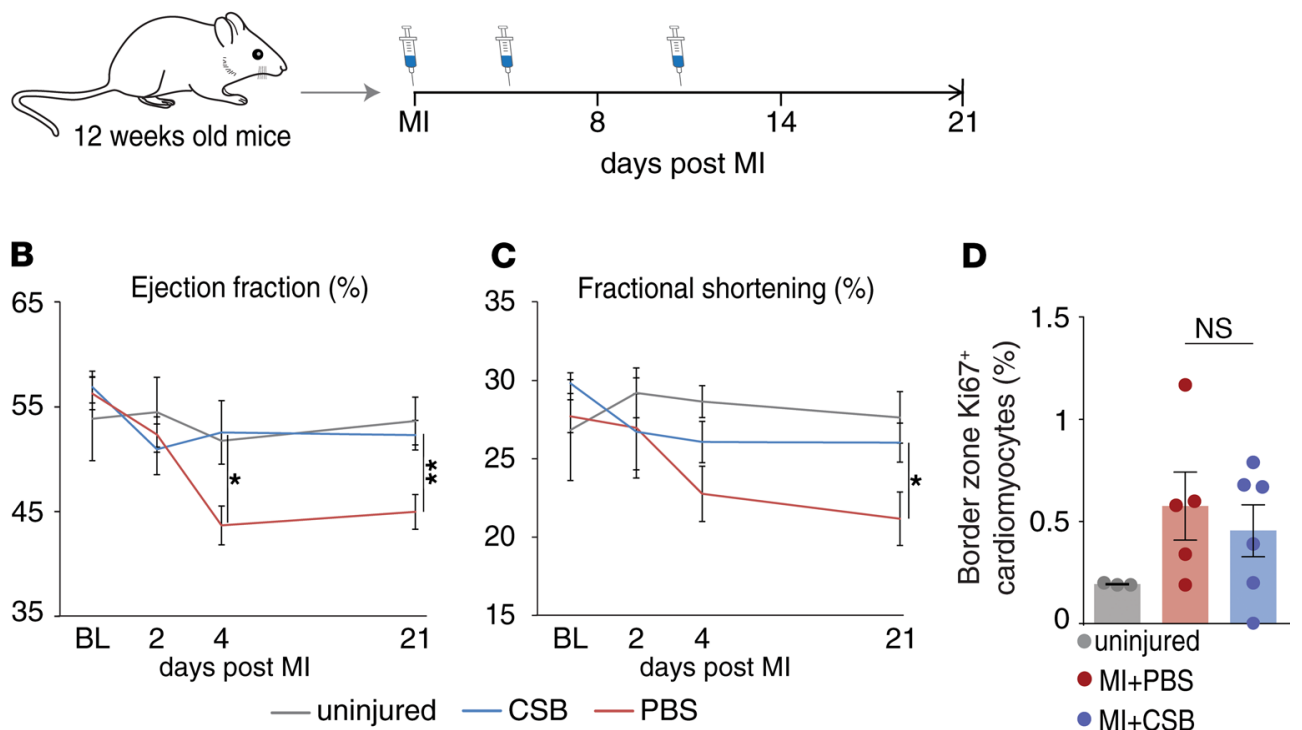

Figure 3. CSB improves cardiac function of adult mice following MI without inducing cardiomyocyte proliferation. (A) Schematic representation of the MI experiment timeline. (B) Cardiac ejection fraction (EF) of uninjured mice $(n=$ 5), PBS-treated mice $(n=6)$, and CSB-treated mice $(n=9)$ up to 21 days after MI (mean \pm SEM). (C) Cardiac fractional shortening (FS) of uninjured mice ( $n=7)$, PBS-treated mice, $(n=8)$, and CSB-treated mice $(n=8)$ up to 21 days after MI (mean \pm SEM). Statistical analysis for $\mathbf{B}$ and $\mathbf{C}$ was performed using 1-way ANOVA and Sidak's correction. Asterisks represent the differences between PBS and CSB treatments. (D) Percentage of Ki67 ${ }^{+}$cardiomyocytes normalized to total cardiomyocytes in the heart of uninjured adult mice $(n=3)$ or in the border zone of PBS-treated $(n=5)$ or CSB-treated $(n=6)$ adult mice 4 days after MI (mean \pm SEM, 1-way ANOVA and Tukey's post hoc test). For all panels: ${ }^{*} P<0.05,{ }^{* *} P$ $<0.01$. NS, not significant; BL, baseline.

as the change in fluorescence intensity relative to baseline $-\mathrm{df} / \mathrm{f}$, at 2- to $5-\mathrm{Hz}$ pacing rate) compared with untreated cardiobundles (Figure 5, B, D, and E). Furthermore, CSB-treated cardiobundles exhibited enhanced contractile strength (as evidenced by higher twitch amplitude; Figure 5, C and F) and faster force generation kinetics (as evident from the shorter twitch duration at 2-Hz pacing, Figure 5G). Such improved calcium handling and contractile force generation in cardiomyocytes treated with CSB may counteract the functional deterioration resulting from ischemic injury in vivo.

Overall, our findings reveal that CSB reduces phospho-CaMKII levels in P8 cardiac cells and engineered 3D cardiac tissues in vitro and in adult mouse cardiomyocytes after MI in vivo. Similarly to CSB, direct CaMKII inhibition by KN62 induces proliferation of $\mathrm{P} 8$ cardiomyocytes in vitro and improves heart function of adult mice after MI. These data suggest that CaMKII inhibition is part of the mechanism by which CSB improves heart function after MI, potentially by improving intracellular calcium handling and force generation, as shown in the cardiobundle system.

CSB inhibits cardiac neutrophil and macrophage activation. In addition to improved cardiac function, CSB reduced scar size in adult mice following MI. These observations led us to examine whether CSB affects the immune response after injury. Numerous cell types participate in the inflammatory process in the heart after injury. These cells communicate with each other in order to recruit more naive immune cells to the injured site, which are in turn activated to execute the inflammatory process. This intercellular communication is performed mainly by secretion of cytokines. To test whether CSB has an effect on the immune response we first tested cytokine levels. We induced MI in adult mice and injected them with CSB or PBS as described above. We evaluated cytokine levels by ELISA 1, 4, or 8 days after MI. Overall, injured mice that were treated with CSB had lower cytokine levels compared with PBS-treated mice (Figure 6, A and C). This was most evident 4 days after MI, when the levels of 12 tested cytokines in CSB-treated mice were equal to those of uninjured mice and lower than those of PBS-treated mice (Figure 6, A and C). In contrast, 4 days after injury the levels of IL-6, MCP-1 (CCL2), MIP-1 $\alpha$ (CCL3), and RANTES (CCL5) were equal or higher after CSB treatment in comparison with PBS (Figure 6, A and B). Interestingly, 3 of these cytokines are chemokine (C-C motif) ligands (CCLs), which promote recruitment of immune cells from 


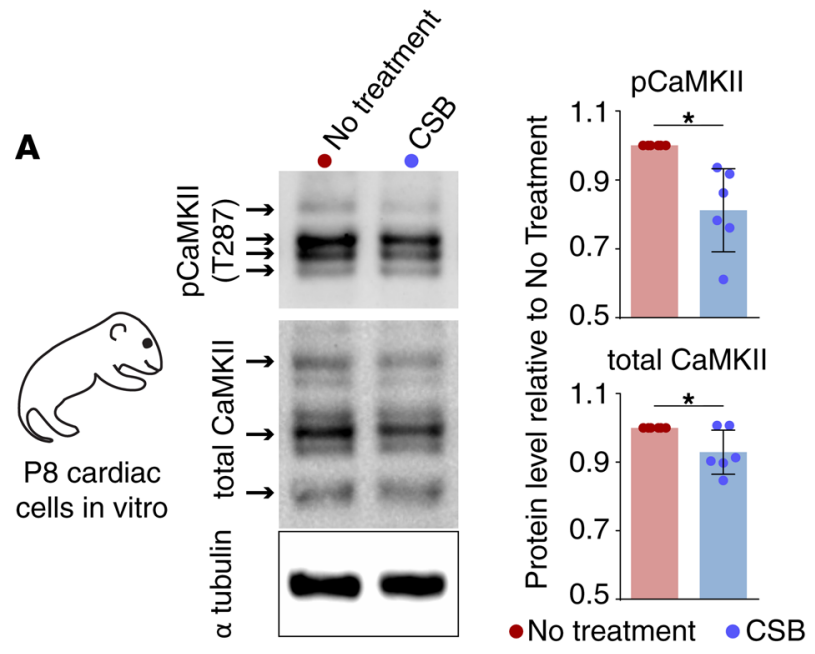

C
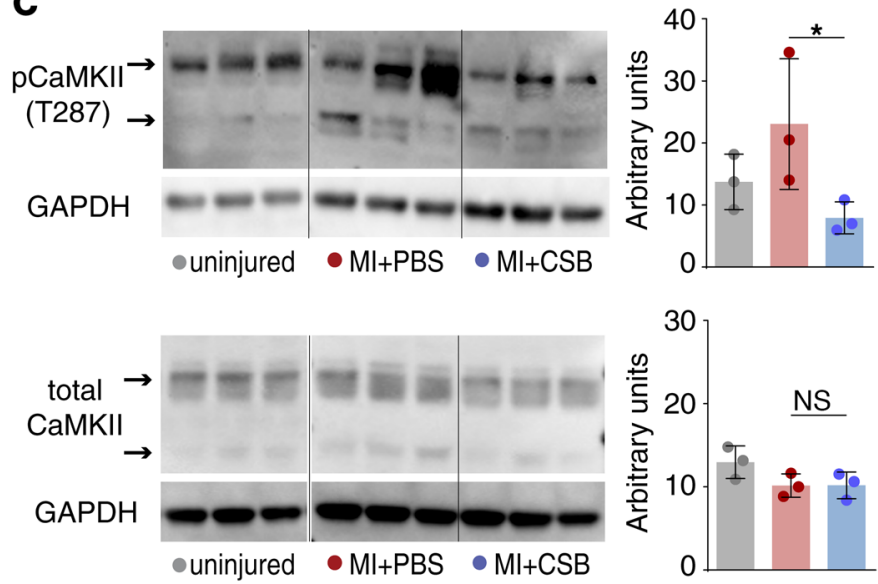

B Number of cardiomyocytes in live culture

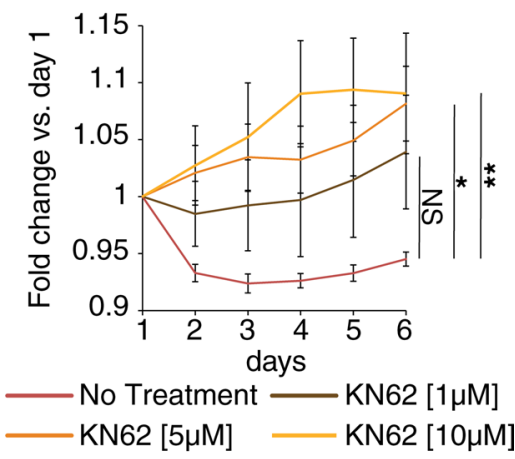

D
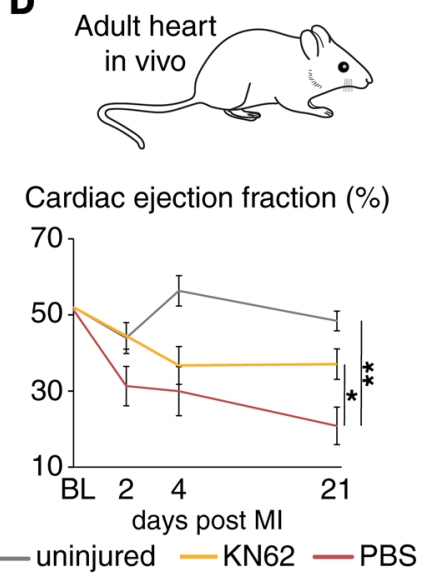

Figure 4. CaMKII inhibition by CSB improves heart function after MI. (A) Western blot images and quantification show the protein level of total CaMKII and phospho-CaMKII (pCaMKII) in P8 cardiac cell cultures treated with CSB for 4 days versus untreated control ( $n=6$ for each group, mean \pm SD, paired 2-tailed Student's $t$ test). (B) Number of P8 cardiomyocytes in live culture relative to day 1 as quantified by the screening system (no treatment, $n=11 ; 1$

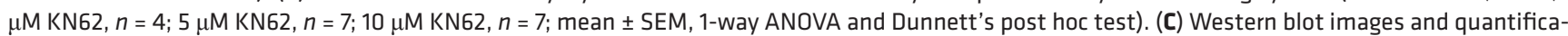
tions show the protein level of total CaMKII (lower panel) and pCaMKII (upper panel) in the ventricles of adult mice 5 days after MI ( $n=3$ for each group, mean \pm SD, 1-way ANOVA and Sidak's correction). Samples from uninjured mice were run in the same gel as MI + PBS and MI + CSB but were noncontiguous. (D) Cardiac EF of adult mice uninjured $(n=3)$ or injured and treated with PBS $(n=6)$ or KN62 $(n=6)$ up to 21 days after MI (mean \pm SEM, 1 -way ANOVA and Dunnett's post hoc test). For all panels: ${ }^{*} P<0.05,{ }^{* *} P<0.01$. NS, not significant.

the blood stream and remote parts of the tissue to the site of the injury. Higher levels of CCLs and lower levels of the other cytokines suggest that CSB does not inhibit the recruitment of immune cells but rather the activation of the recruited cells at the site of injury, which may result in attenuated inflammation.

To further test this hypothesis, we used fluorescence-activated cell sorting (FACS) to quantify the number of immune cells in the hearts of 12-week-old mice following injury. Four days after MI, CSB treatment did not change the number of total leukocytes $\left(\mathrm{CD} 45^{+}\right)$, myeloid lineage cells $\left(\mathrm{CD} 45^{+}, \mathrm{CD} 11 \mathrm{~b}^{+}\right)$, total neutrophils $\left(\mathrm{CD}^{2} 5^{+}, \mathrm{CD} 11 \mathrm{~b}^{+}, \mathrm{Ly}_{6 \mathrm{G}}{ }^{+}\right)$, or total macrophages $\left(\mathrm{CD} 45^{+}, \mathrm{CD}_{11 \mathrm{~b}}, \mathrm{~F} 4 / 80^{+}\right.$, $\left.\mathrm{Ly}_{6 \mathrm{G}}\right)$ (Figure 7, A-C, and E), indicating that CSB does not inhibit the recruitment of these cells. The C-C chemokine receptor type 2 (CCR2) is a chemotaxis receptor expressed on naive neutrophils and macrophages. Binding of CCL chemokines to CCR2 leads to the recruitment of the CCR2-expressing cells from the periphery to the site of the injury, where they are activated. During the activation phase CCR2 is downregulated and the cells become CCR2- (58). Examination of the neutrophil and macrophage populations revealed a higher fraction of CCR2 ${ }^{+}$cells in CSB-treated hearts (Figure 7, D and F). The fact that CSB increased the fraction of $\mathrm{CCR} 2^{+}$cells without changing the total number of cells supports the idea that CSB does not inhibit cell recruitment but rather their activation at the site of injury, thereby attenuating the inflammatory response. 
A

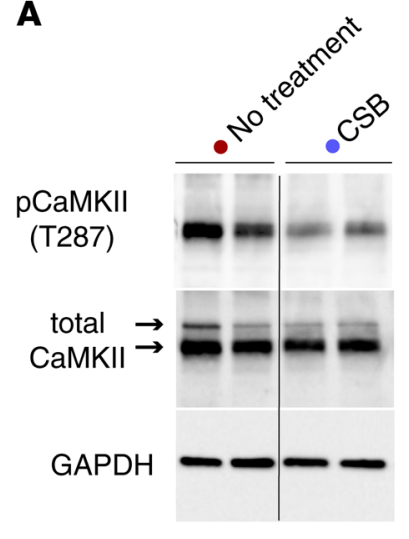

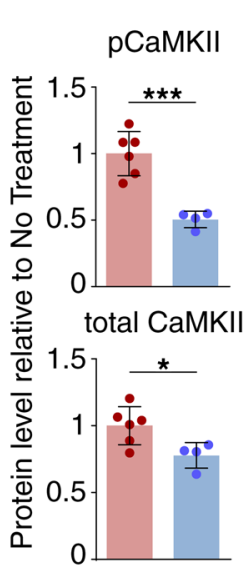

B $1.5 \quad 5 \mathrm{~Hz} \mathrm{Ca}^{2+}$ trace

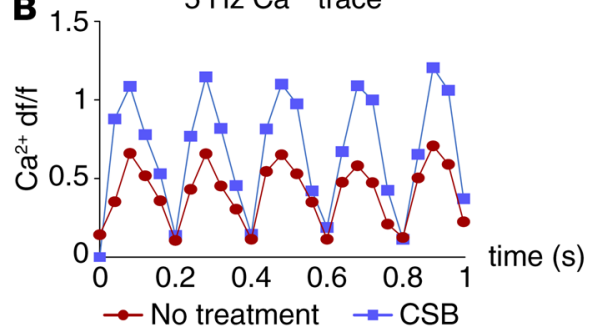

C $5 \mathrm{~Hz}$ Active Force

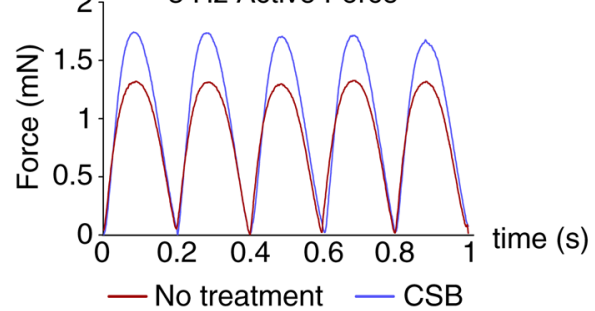

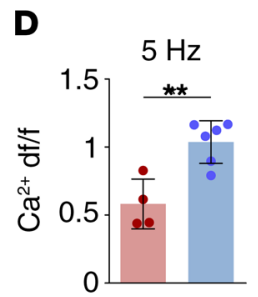
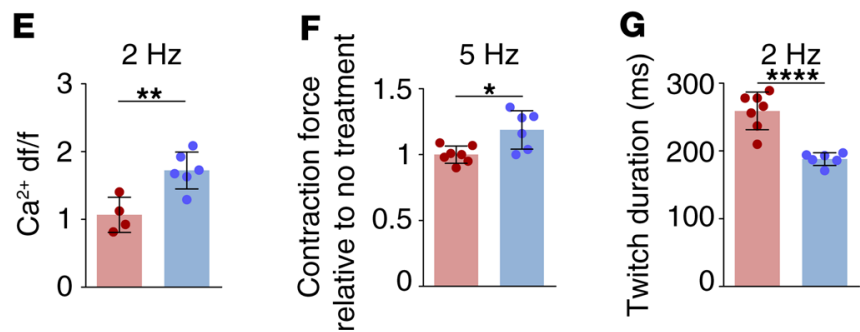

- No treatment

- CSB

Figure 5. CSB improves cardiomyocyte calcium handling and contraction in NRVM cardiobundles. (A) Representative Western blots and quantification showing decreased protein levels of total CaMKII and phospho-CaMKII (pCaMKII) in cardiobundles treated with CSB versus no treatment (no treatment, $n=6$; CSB, $n=4$; mean \pm SD, unpaired 2-tailed Student's $t$ test). Data were obtained from 2 separate NRVM isolations. (B) Representative calcium transients in cardiobundles during 5- $\mathrm{Hz}$ pacing recorded by GCaMP6 sensor (shown as change in fluorescence intensity relative to baseline, df/f). (C) Representative isometric twitch force traces in cardiobundles during $5-\mathrm{Hz}$ pacing. (D and E) Calcium df/f in cardiobundles paced at $5 \mathrm{~Hz}$ (D) or $2 \mathrm{~Hz}$ (E) (no treatment: $5 \mathrm{~Hz} n=4,2 \mathrm{~Hz} n=4$; CSB: $5 \mathrm{~Hz} n=6,2 \mathrm{~Hz} n=6$; mean \pm SD, unpaired 2-tailed Student's $t$ test). (F) Twitch amplitude (max force) in cardiobundles paced at $5 \mathrm{~Hz}$ treated with CSB versus untreated control; data presented relative to no treatment (no treatment, $n=7 ; \mathrm{CSB}, n=6 ;$ mean \pm SD, unpaired 2 -tailed Student's $t$ test). Data were obtained from 2 separate NRVM isolations. (C) Twitch duration in CSB-treated cardiobundles versus untreated control paced at $2 \mathrm{~Hz}$ (no treatment, $n=7 ; C S B, n=6$; mean \pm SD, unpaired 2-tailed Student's $t$ test). Data were obtained from 2 separate NRVM isolations. In all cardiobundle experiments CSB was administered at a concentration of $50 \mu \mathrm{M}$. For all panels: ${ }^{*} P<0.05,{ }^{* *} P<0.01,{ }^{* *} P<0.001,{ }^{* * * *} P<0.0001$.

To further test this, we stained the hearts for Mac2 (also known as galectin-3), a protein that is expressed on activated macrophages in failure-prone hearts (59). Mac2 promotes cardiac fibroblast proliferation and collagen production, which leads to ventricular dysfunction (59). To test changes in Mac2 expression levels, we first compared the area of dead tissue in CSB- and PBS-treated hearts 2 and 4 days after MI as an indication of the initial damaged induced by the MI and found no significant difference (Figure 7, G and H). Although there was no significant difference in the initial size of the injury, CSB-treated mice exhibited significantly lower levels of Mac2 staining 4 and 8 days after MI, reflecting the lower level of activated macrophages (Figure 7, I and J). The mechanism by which CSB inhibits cardiac neutrophil and macrophage activation requires further investigation. However, we found that CSB administration can modulate inflammatory gene expression in cultures of pure bone marrow-derived macrophages (BMDMs) (Supplemental Figure 3). This suggests that CSB affects macrophages directly and not necessarily through its effect on cardiomyocytes.

Altogether, our data show that CSB does not inhibit the recruitment of the immune cells but rather the activation of neutrophils and macrophages in the heart after MI. This inhibition may lead to the attenuated inflammation and thereby modulation of the scar.

\section{Discussion}

In this study we demonstrate a therapeutic potential of the small molecule CSB that suppresses detrimental processes following cardiac injury and facilitates repair by improving cardiomyocyte contractility and attenuating the immune response (Figure 8). Small molecules are promising therapeutic candidates for 
A
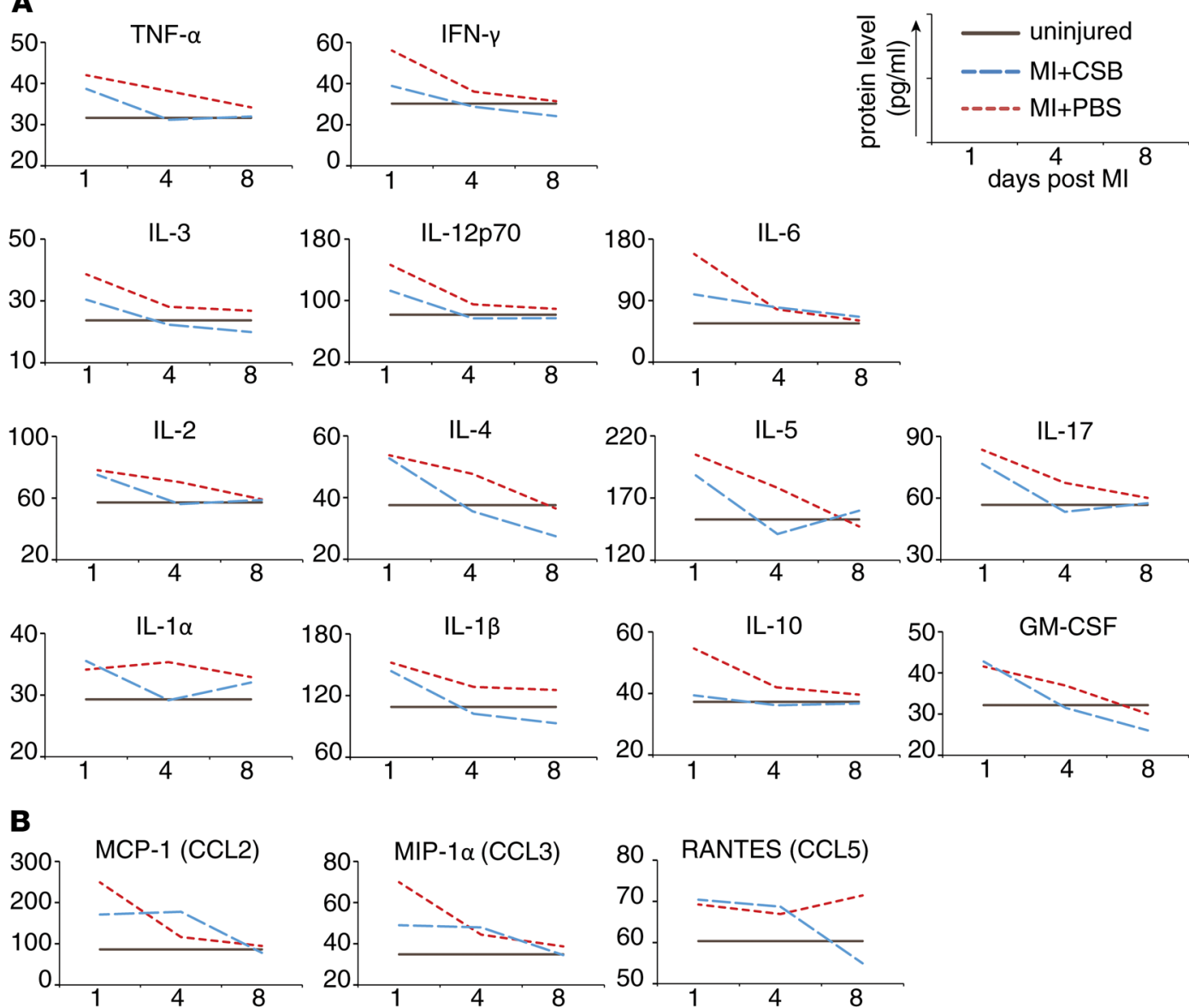

C

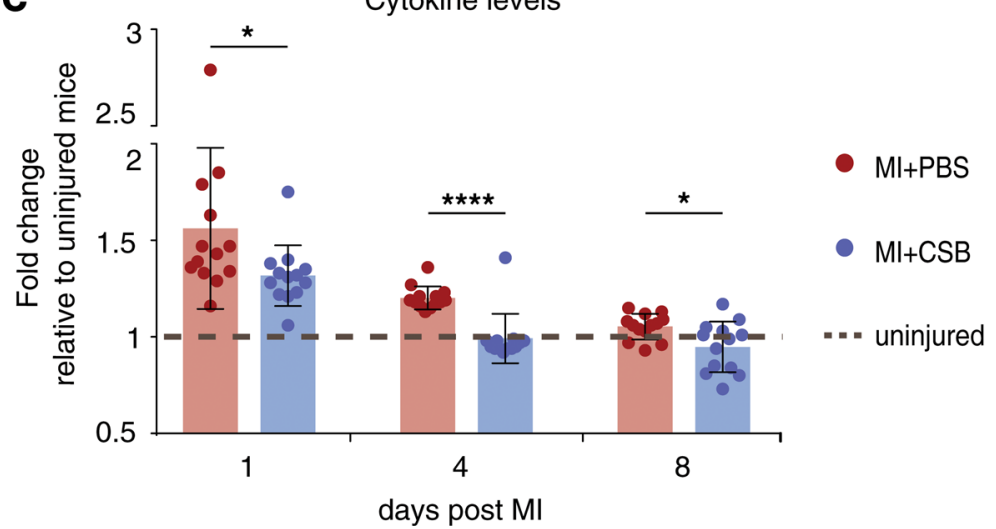

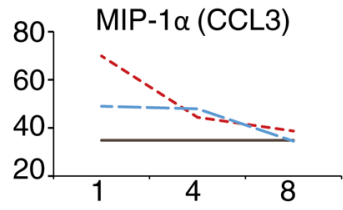

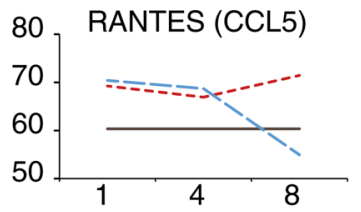

Figure 6. CSB inhibits immune cell activation but not recruitment. Twelveweek-old mice underwent $\mathrm{MI}$ and were injected with CSB or PBS. The first injection was i.m. into the myocardium immediately after injury. Additional i.v. injections were given 2, 4, and 6 days after MI. Hearts were harvested 1,4 , or 8 days after MI and ELISA was performed. All tested cytokines are presented. (A) Cytokine levels show general attenuation of the inflammatory response after CSB treatment. (B) The level of the 3 tested chemokine (C-C motif) ligands (CCLs) is not inhibited by CSB 4 days after MI. (C) Average change in the levels of all cytokines except CCLs (cytokines in panel A) relative to uninjured mice. For each treatment at each time point $n$ $=13$, mean \pm SEM, paired 2-tailed Student's $t$ test. ${ }^{*} P<0.05$, ${ }^{* * *} P<0.0001$.

several reasons. They are easy and inexpensive to produce and store, easy to administer, allow temporal control of their targets, and are tolerated by the immune system. Therefore, we developed a high-throughput screening system for small molecules in cultured P8 cardiomyocytes with the readout of cardiomyocyte cell number. The rationale behind our screen was to avoid cell cycle activity markers that could be misleading $(30,31)$ by focusing on changes in cardiomyocyte number per well. Because P8 cardiomyocytes do not proliferate extensively the assay is subjected to high noise and many repeats are required in order to obtain a positive hit. One such hit identified in this screen was CSB, a multifunctional molecule used mostly for its blue color, for example, as a counterstain to reduce autofluorescence background in immunofluorescence staining, for eye lens and cornea staining (60), and for staining of lymph nodes (61). CSB was shown to have several biological targets, including VGLUT (46), MIF (38), CaMKP (62), RAD51 (39), ubiquitin (40), and the Prion protein (63). In vivo, CSB was shown to attenuate methamphetamine-induced hyperac- 

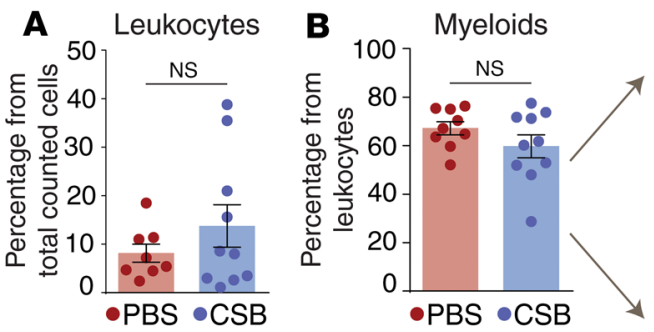

G Area of Injured tissue

2 days post MI
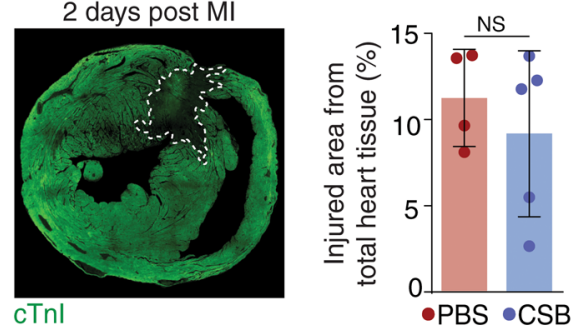

H
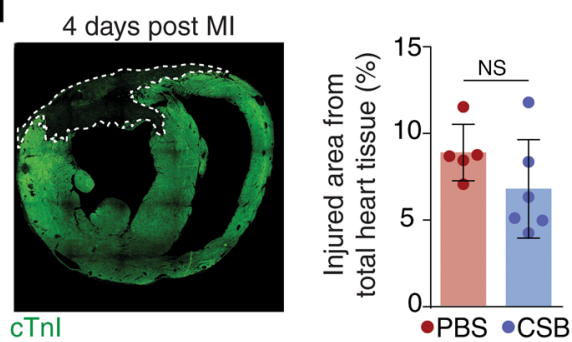

I

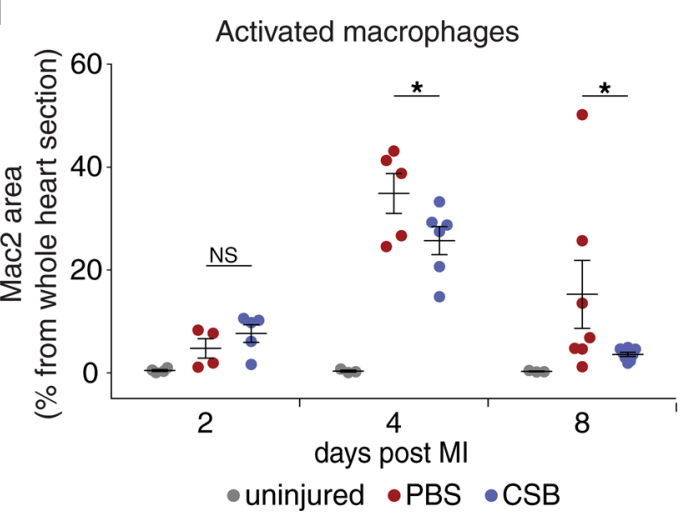

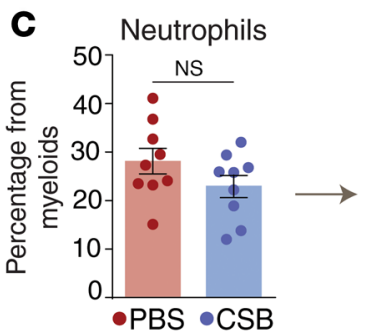
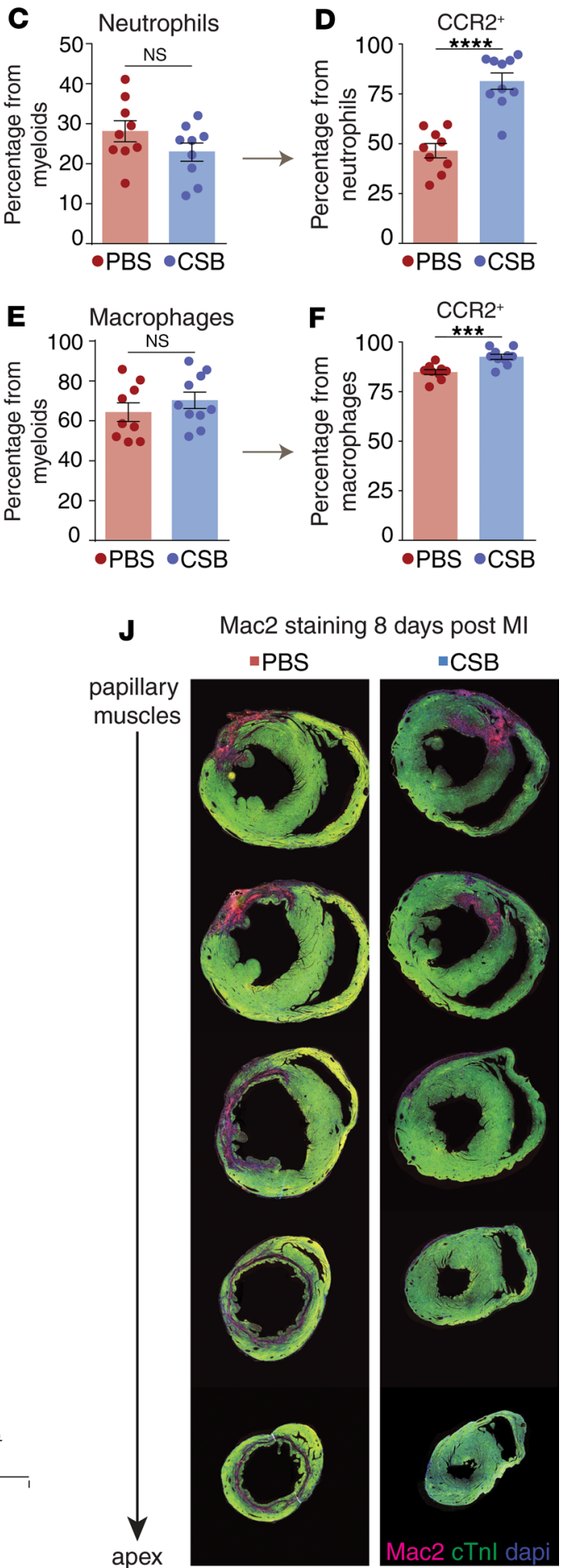

Figure 7. CSB inhibits neutrophil and macrophage activation in the heart following MI. (A-C and E) FACS analysis shows the percentage of total leukocytes (A), myeloid cells (B), neutrophils (C), and macrophages (E) in the heart of adult mice 4 days after MI. (D and F) Percentage of CCR2 ${ }^{+}$neutrophils (D) and macrophages (F) in the hearts of adult mice 4 days after MI. Results for $\mathbf{A}-\mathbf{F}$ were obtained from 2 separate experiments. PBS, $n=9 ;$ CSB, $n=10 ;$ data presented as mean \pm SEM, unpaired 2-tailed Student's $t$ test. (G-J) Mac2 staining in injured hearts. Adult mice underwent MI and were treated with PBS or CSB in the same manner as in the ELISA experiment (1 i.m. and 3 i.v. injections). Hearts were isolated 2, 4, and 8 days after MI and stained for cardiac troponin I (cTnl) as a cardiomyocyte marker and Mac2. (G) Representative image of injured heart 2 days after MI and quantification of the injured zone size in PBS-treated $(n=4)$ or CSB-treated $(n=5)$ mice (mean \pm SD). (H) Representative image of injured heart 4 days after MI and quantification of the injured zone size in PBS-treated $(n=5)$ or CSB-treated $(n=6)$ mice (mean \pm SD). (I) Quantification of activated macrophages in the hearts of adult mice 2 days (uninjured, $n=4$; PBS, $n=4$; CSB, $n=5$ ), 4 days (uninjured, $n=3$; PBS, $n=5 ;$ CSB, $n=6$ ), and 8 days (uninjured, $n=3 ;$ PBS, $n=7$; CSB, $n=8$ ) after MI. Quantification was performed by measuring the area stained for Mac2 as percentage of the area of the whole section (mean \pm SEM). (J) Representative serial sections of adult mouse hearts stained for Mac2 8 days after MI. Statistical analysis for G-I was performed using 1-tailed, unpaired Student's $t$ test. For all panels: ${ }^{*} P<0.05,{ }^{* * *} P<0.001,{ }^{* * *} P<0.0001$. NS, not significant. 


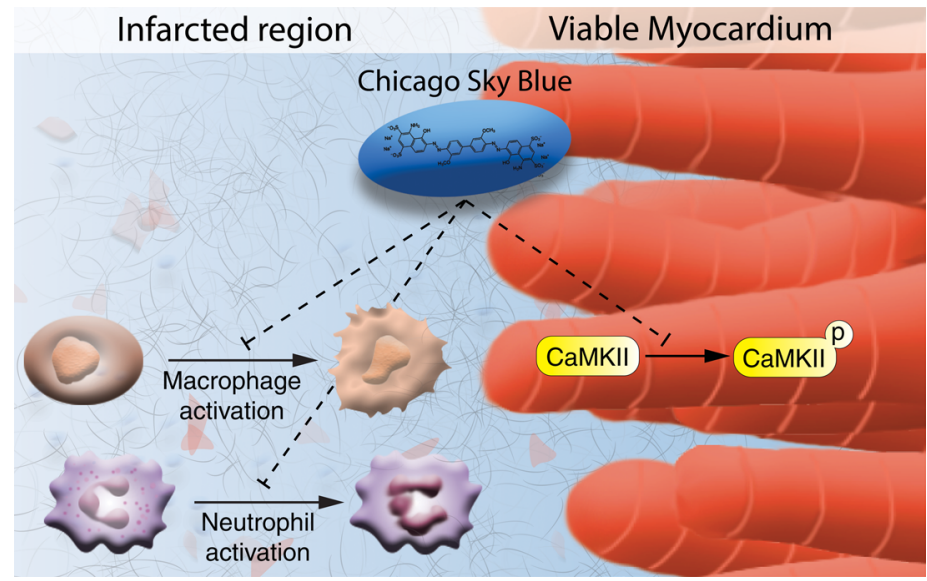

Figure 8. Suggested mechanism of action utilized by CSB to promote heart repair following MI. CSB promotes heart repair after MI through inhibition of neutrophil and macrophage activation that attenuates the acute inflammatory response and through inhibition of CaMKII signaling, which improves cardiomyocyte contractility.

tivity (46), to have antinociceptive effects (44), to attenuate secondary injuries in the spinal cord (45), and to modulate feeding in broilers (42).

Among its known targets, the most established and studied function of CSB is the inhibition of VGLUT, which is expressed mainly in the nervous system and facilitates the secretion of glutamate as a neurotransmitter $(37,64)$. Although we observed Vglut RNA expression in cardiomyocytes, we could not induce P8 cardiomyocyte proliferation using other VGLUT inhibitors. In addition, we tested multiple glutamate receptor agonists (NMDA, AMPA, kainic acid, ATPA, ACPD, DHPG, L-AP4) and antagonists (GYKI, CNQX, MK-801, S-MCPG) and again failed to observe induction of P8 cardiomyocyte proliferation (data not shown).

One of the mechanisms by which CSB facilitates heart repair after MI is the inhibition of CaMKII activity, which was shown to have deleterious effects on the heart following injury, including adverse cardiac remodeling $(65,66)$ and impaired cardiomyocyte contraction $(35,36)$. Direct CaMKII inhibition or examination of CaMKII-knockout mice revealed cardiac protection after MI $(35,36)$. Our results suggest that CaMKII inhibition by CSB is likely indirect. During the first 2 days of screening for cardiomyocyte number we could not detect an effect of CSB. Hence, quantification of cardiomyocyte number was relative to day 2 (Figure 1B). When we treated cardiac P8 cells with the CaMKII inhibitor KN62 we noticed the effect earlier (on day 1, Figure 4B) compared with CSB, suggesting that there are intermediate signaling steps between the 2 processes. In addition, we did not observe changes in CaMKII or phospho-CaMKII levels in non-cardiomyocytes treated with CSB, implying that CaMKII inhibition does not involve direct binding of CSB to CaMKII (Supplemental Figure 2).

Another mechanism by which CSB facilitates heart recovery following MI is by attenuation of the immune response. Following cardiac injury the immune response can be either essential for the repair (67-69) or harmful for the process (70-72). Yet, there is broad agreement that a complete inhibition of the immune response, or its extensive and prolonged activation, is detrimental for heart repair (73-75). The results from the FACS analysis and Mac2 staining suggest that CSB partially inhibited neutrophil and macrophage activation. Because the ELISA results show an increase in cytokine levels in injured hearts 1 day after MI in mice treated with CSB, it suggests that the initial inflammatory phase was unperturbed but the later resolution phase, in which scarring takes place, was suppressed. This moderate attenuation of the immune response in the heart might shift the balance from deterioration to repair.

Recent studies describe a population of tissue-resident CCR2 ${ }^{-}$macrophages that are required for regeneration of neonatal mouse heart but exist in very low numbers in the nonregenerative adult heart (76). Our interpretation of the results describing CSB's effect on the number of CCR2- macrophages suggests that CSB protects the heart by inhibiting macrophage maturation and thus maintains CCR2 expression as described in Phillips et al. (58). A deeper characterization of the influence of CSB on distinct subpopulations of immune cells is required to better understand its mode of action.

An attenuated immune response could lead to reduced fibrosis. Previous work showed that CSB decreases fibrosis and attenuates secondary injuries following spinal cord injury (45). Although we observed distinct reduction in scar size 8 days after MI following CSB treatment, the reduction in scar size 21 days after MI was milder. Furthermore, we were not able to detect a significant reduction in collagen gene expression (Colla1 and Col3a1) or fibroblast levels in injured hearts following CSB treatment (data 
not shown). This raises the possibility that the attenuated immune response induced by CSB can induce multiple mechanisms to promote heart repair after MI other than reduced fibrosis, e.g., by prevention of detrimental secondary responses or high apoptosis that occur in the first days following MI (74, 77-79).

We postulate that CSB treatment improves the recovery of the injured heart by 2 different inhibitory mechanisms affecting CaMKII signaling in cardiomyocytes and the inflammatory response. Several studies implicate CaMKII activity in cardiomyocytes with adverse inflammatory response following MI $(80,81)$, or angiotensin II infusion (50), and CaMKII knockout in cardiomyocytes with attenuated inflammation. Whether CSB affects these 2 processes in parallel or whether the observed attenuation of the immune response is mediated via CaMKII inhibition in cardiomyocytes is yet to be determined.

Although CSB was identified using a screen for P8 cardiomyocyte proliferation in vitro, in adult mice CSB does not seem to induce cardiomyocyte proliferation. This discrepancy was observed in several other studies describing compounds that induce neonatal cardiomyocyte proliferation and adult heart regeneration. These studies reported a low cardiomyocyte proliferation rate in the regenerated adult heart, suggesting that additional mechanisms are involved in the regeneration and repair process, e.g., neuregulin 1 (26, 82 ) or agrin $(23,83)$. A possible explanation for this discrepancy is that activation or inhibition of a certain cellular component or pathway is age dependent (84). Overall, our cumulative findings suggest that cardiac repair processes can be boosted in the adult mouse heart even in the absence of cardiomyocyte renewal.

From a clinical point of view, we did not find evidence of toxicity induced by CSB after 3 weeks of treatment. It will be interesting to test CSB efficacy in large animals and using different administration methods and to define the time window after injury in which CSB treatment would be most effective. Several studies have attempted to inhibit CaMKII $(35,36,47,51,85)$ or the inflammatory response $(70,72$, $75,86,87)$ in order to augment heart repair after MI; however, safe and effective drugs are yet to be discovered. Our study highlights another strategy for improving cardiac repair by simultaneously attenuating the immune response and improving cardiomyocyte contractility.

\section{Methods}

Animals. The mice that were used for the high-throughput screen and in vitro immunofluorescence staining were generated by crossing Myh6-Cre mice (88) with Rosa26-tdTomato mice (89) (MHC:Tomato). Myh6-Cre mice harbor a Cre-coding sequence inserted after the Myh6 promotor, which drives high-efficiency gene recombination specifically in cardiomyocytes. Rosa26-tdTomato mice have a conditional red fluorescent protein (RFP) allele that requires CRE-mediated recombination for expression. This system allowed visualization of RFP-labeled cardiomyocytes in a mixed cardiac cell culture. Rosa26-tdTomato and Myh6-Cre mice were maintained on the background of C57BL/6J mice. No sex selection was performed for these experiments. For in vivo MI surgery experiments, 12-week-old Hsd:ICR female mice (Envigo RMS) were used. For purification of BMDMs, 8-week-old C57BL/6JOlaHsd female mice were used.

Cardiac cell isolation. Primary cardiac cells were isolated from P8 MHC:Tomato mice or adult 12-weekold mice using a neonatal cardiac cell dissociation kit (gentleMACS, Milteny Biotec) according to the manufacturer's instructions.

High-throughput screening. Cardiac cells were isolated from P8 MHC:Tomato mice and were homogeneously suspended in plating medium (DMEM/Ham's F12) supplemented with L-glutamine (1\%), pyruvate $(1 \%)$, nonessential amino acids (1\%), penicillin/streptomycin (1\%), horse serum (5\%), and fetal bovine serum (FBS) (20\%). The cells were equally plated on 384-well optical-bottom plastic plates precoated with gelatin ( $0.02 \%$ gelatin, G1393, Sigma-Aldrich) at a concentration of 1000 cardiomyocytes per $50 \mu \mathrm{L}$ medium per well. Twenty-four hours after plating, the plating medium was aspirated using GNF Systems WDII and replaced with growing medium composed of the same ingredients as the plating medium except FBS. Immediately after medium replacement, each well was introduced to a different small molecule using the Agilent Bravo automated system. All small molecules were dissolved in DMSO/double-distilled water (DDW) to a final concentration of $10 \mu \mathrm{M}$. Relevant control wells were also included. Twenty-four hours after compound administration (day 1) the TTP Labtech Acumen system was used to image each well under fluorescence excitation, to detect only tdTomato-expressing cardiomyocytes. The wells were imaged daily for 6 days. Using Cellista software, the number of cardiomyocytes in each well was counted based on cell segmentation. A graph of the number of cardiomyocytes in each well relative to the number of cardiomyocytes in the same well at the beginning of the experiment was generated using Accelrys Pipeline Pilot software. On the first imaging day, 24 hours after compound administration, each well contained 200-300 
cardiomyocytes. During the experiment the plates were incubated at $37^{\circ} \mathrm{C}$ and $5 \% \mathrm{CO}_{2}$.

In vitro cell culture. For P8 MHC:Tomato cardiac cells: primary cardiac cells were isolated, homogeneously suspended in plating medium, plated on gelatin-coated wells, and cultured as described above. The cells were plated at a concentration of 10,000 cardiomyocytes in $200 \mu \mathrm{L}$ medium per well in 96 -well plates. The cells were allowed to adhere for 24 hours; subsequently, the medium was replaced with growing medium (described above) containing the investigated molecules. Growing medium was exchanged every other day.

For adult 12-week-old non-cardiomyocytes: hearts were isolated and cut to small pieces, digested using the neonatal cardiac cells dissociation kit (gentleMACS, Milteny Biotec) according to the manufacturer's instructions, and were plated in gelatin-coated 6-well plates. Cells from each heart were plated in 2 wells (of a 6-well plate) - $2 \mathrm{~mL} /$ well, in BIO-AMF-2 (Biological Industries, 01-194-1A). Six hours after plating, cells were washed 3 times with BIO-AMF-2 and incubated at $37^{\circ} \mathrm{C}$ for 4 days. Each day the cells were washed 3 times with BIO-AMF-2. Four days after plating the cells were detached from the plate using trypsin and were plated on new gelatin-coated 6-well plates in fibroblast plating medium (DMEM/Ham's F12) supplemented with horse serum (5\%), L-glutamine (1\%), pyruvate $(1 \%)$, nonessential amino acids $(1 \%)$, penicillin/streptomycin (1\%), and FBS (10\%) at $3 \mathrm{~mL} /$ well. This passaging process was repeated 3 days later (7 days after plating). At this point the culture did not contain cardiomyocytes. Eight days after plating the fibroblast plating medium was replaced with fibroblast growing medium (composed of the same ingredients as the fibroblast plating medium without FBS), with or without $10 \mu \mathrm{M} \mathrm{CSB}, 2 \mathrm{~mL} /$ well. Growing medium was exchanged every other day.

Cell culture immunofluorescence staining. Cultures of MHC:Tomato mouse cardiac cells were fixed with 4\% paraformaldehyde in PBS for 15 minutes and washed; permeabilized with $0.5 \%$ Triton X-100 in PBS for 10 minutes and washed; blocked with PBS containing $5 \%$ bovine serum albumin (BSA) and $0.1 \%$ Triton X-100 for 1 hour; all at room temperature. The cells were incubated overnight at $4^{\circ} \mathrm{C}$ with rabbit anti-Ki67 (SP6) antibody (1:200, Cell Marque, 275R) diluted in blocking solution. Cells were then washed with PBS and incubated for 45 minutes at room temperature with suitable secondary antibody and DAPI (4',6-diamidino-2-phenylindole) diluted in blocking solution. Cells were imaged using a Nikon Eclipse Ti2 or Olympus live cell imaging microscope.

MI (LAD ligation). MI was induced in 12-week-old mice by ligation of the LAD coronary artery. Mice were sedated in an isoflurane box (Abbott Laboratories) followed by tracheal intubation. Following skin incision, lateral thoracotomy at the third intercostal space was performed by blunt dissection of the intercostal muscles. LAD ligation was performed using 8-0 suture. Following artery ligation, CSB or PBS was injected intramyocardially. Next, the skin was closed with 6-0 nonabsorbable silk suture or skin-adhesive glue. Mice were then warmed for several minutes until recovery.

Heart tissue sectioning and staining. Hearts were isolated and fixed in 4\% paraformaldehyde in PBS for 3-7 days. Next, the hearts were embedded in paraffin, sectioned transversely at $5-\mu \mathrm{m}$ thickness in $400-\mu \mathrm{m}$ intervals, and loaded on a slide. The sections underwent deparaffinization with xylene and a decreasing ethanol gradient. For histochemistry, sections were stained with H\&E or Masson's trichrome. For immunofluorescence, sections underwent microwave antigen retrieval in citrate buffer ( $\mathrm{pH}$ 6.0) or EDTA buffer, followed by gradual cooling to room temperature. Samples were permeabilized and blocked with PBS containing 20\% horse serum and $0.5 \%$ Triton X-100 for 1.5 hours at room temperature. Next, samples were incubated in a wet chamber overnight at room temperature with the following primary antibodies diluted in PBS containing 2\% horse serum and 0.5\% Triton X-100: mouse anti-cardiac troponin T (cTnT) (1:200, Abcam, ab33589); rabbit anti-Ki67 (SP6) (1:200, Cell Marque, 275R); rat anti-Mac2 (1:250, Cedarlane, CL8942AP); and rabbit anti-cardiac troponin I (cTnI) (1:200, Abcam, ab47003). Next, the sections were washed and incubated with secondary antibodies and DAPI for 1 hour at room temperature. Slides were mounted with immune-mount (9990412, Thermo Fisher Scientific) and imaged using a Nikon Eclipse Ti2 or Olympus live cell imaging microscope. For DAB immunohistochemistry, the sections were bleached with methanol, $1 \% \mathrm{H}_{2} \mathrm{O}_{2}$, and $0.3 \% \mathrm{HCl}$ for 30 minutes. Heat-mediated antigen retrieval was done using citrate buffer ( $\mathrm{pH}$ 6.0). The sections were incubated in $20 \%$ horse serum and $0.2 \%$ Triton X-100 in PBS for permeabilization and blocking of nonspecific binding and then incubated with rat anti-Mac2 antibody (1:500, Cedarlane, CL8942AP) overnight. Biotinylation was performed using biotin-conjugated donkey anti-rat antibody (Jackson Laboratories, 712066153). Signal amplification was done with avidin-biotin complexes (Vector laboratories, VECTASTAIN Elite ABC HRP Kit, PK-6100). One hundred and twenty microliters of DAB mixture was loaded on each slide. The DAB- 
stained slides were counterstained with hematoxylin. Slides were dehydrated with an increasing gradient of ethanol and were mounted with Entellan (xylene-based mounting) (Sigma-Aldrich).

Infarct size quantification. Measurements were done on transverse heart sections. To quantify infarction size, a dot was marked in the middle of the left ventricle and 2 lines were stretched from the dot to the infarcted border in the section. One line pointed to one end of the infarct zone - between the infarcted zone and the healthy tissue, and a second line pointed to the other end of the infarct. The size of infarcted area was calculated as: (the angle between the 2 lines) $/ 360 \times 100$. For each heart, the infarcted area was measured in 5 sequential sections located between the ligation site and the apex.

Echocardiography measurements and analysis. Echocardiography measurements were done using the Vevo 3100 machine, MX550D transducer for Figure 3, B and C, or the Vevo 770 machine (VisualSonic) for Figure $4 \mathrm{D}$ and Supplemental Figure 1B. Mice were sedated using 3\%-3.5\% isoflurane and then placed on a prewarmed ultrasound stage. During the acquisition the mice were continuously exposed to $2 \%$ isoflurane and kept at 400-500 bpm. Echocardiography records were analyzed using the Vevo LAB program. Cardiac EF was calculated as the average EF measured from the long and short axis of the heart. Cardiac FS was measured only on the short axis. Mice were excluded from the analysis if they exhibited less than 5\% decrease in measured variables at all time points, indicative of inefficient MI, or if they exhibited a decrease of more than $55 \%$ in a measured variable 2 days after MI, indicative of too severe injury.

Pathological examination. Mice were sacrificed 21 days after MI following 7 injections of CSB. The first injection was $50 \mu \mathrm{L}$ of $100 \mu \mathrm{M}$ CSB and was injected i.m. into the myocardium. Six additional injections were $100 \mu \mathrm{L}$ of $100 \mu \mathrm{M}$ CSB and were injected i.v. into the tail vein. The last i.v. injection was given 14 days after MI. PBS-treated mice were examined for comparison. Mouse brain and skeletal muscles were isolated. The brain was trimmed in situ into 5-7 slices. Muscles of the caudal thigh and those attached to the outer aspect of the skull were examined. Gross lesions, tumors, or other pathologic changes were not identified. A total of 6 CSB-treated and 5 PBS-treated mice were examined.

Western blot. Western blotting was performed after running samples in 4\%-12\% SDS-PAGE and transferring the proteins to PVDF membranes. The membranes were blocked and incubated at $4^{\circ} \mathrm{C}$ overnight with the following primary antibodies: rabbit anti-CaMKII beta gamma delta (phospho T287) (1:1000, Abcam, ab182647); rabbit anti-CAMKII (1:1000, Abcam, ab52476); mouse anti- $\alpha$-tubulin (1:10,000, Sigma-Aldrich, T5168); and rabbit anti-GAPDH (1:2000, Sigma-Aldrich, PLA0125). Antibodies conjugated to horseradish peroxidase (HRP) were used as secondary antibodies diluted 1:2000. The membranes were incubated with the secondary antibodies for 30 minutes at room temperature. Both primary and secondary antibodies were diluted in Tris-buffered saline with $0.5 \%$ Tween (TBST) containing 5\% skim milk powder or BSA. The membranes were developed using SuperSignal West Pico Chemiluminescent Substrate (Thermo Fisher Scientific, PI34080). All compared samples were run in the same gel and analyzed concomitantly.

Preparation of NRVM cardiobundles, measurements of calcium transients and contractile force. NRVMs were isolated from 2-day-old Sprague-Dawley rats and used to construct tissue-engineered cardiobundles as previously described $(54,90)$. Briefly, hearts were removed and lysed using trypsin and several incubations with collagenase type 2 . The dissociated cells were preplated on tissue-culture plastic to increase the purity of cardiomyocytes. The cells were then mixed in a fibrin-based hydrogel $(2 \mathrm{mg} / \mathrm{mL}$ fibrinogen, $1 \mathrm{U} / \mathrm{mL}$ thrombin, $10 \%[\mathrm{v} / \mathrm{v}]$ Matrigel) and plated in a polydimethylsiloxane (PDMS) tissue mold containing a polymer frame. The cell/hydrogel mixture was allowed to polymerize and attach to the frame at $37^{\circ} \mathrm{C}$ for 45 minutes. PDMS molds with tissues were then cultured dynamically on a rocker in growth medium containing DMEM, horse serum (10\%), chick embryo extract $(1 \%)$, aminocaproic acid $(1 \mathrm{mg} / \mathrm{mL})$, ascorbic acid 2-phosphate sesquimagnesium salt hydrate $(50 \mathrm{mg} / \mathrm{mL})$, penicillin $(5 \mathrm{U} / \mathrm{mL})$, and vitamin B12 $(2 \mathrm{mg} /$ $\mathrm{mL}$ ). On culture day 2, frames with tissues were gently removed from the mold and left to free-float in the medium while cultured dynamically. Growth medium was replaced every other day and calcium transient and contractile force measurements performed on culture day 14.

For calcium transient measurements, NRVMs were transduced by a GCaMP6 lentivirus at the time of cardiobundle formation or stained with Fluo-4 AM (Invitrogen) dye at the time of measurement. Specifically, a lentivirus with muscle-specific promoter MHCK7 driving expression of calcium sensor GCaMP6 (MHCK7GCaMP6) was constructed as previously described (57). Lentiviral concentration was titrated (1:100-1:1000) to allow transduction of approximately $30 \%$ cardiomyocytes and minimize toxicity. For calcium transient measurements with Fluo-4 AM, cardiobundles were washed in Tyrode solution, incubated with 5 nM Fluo-4 AM and $0.02 \%$ pluronic F-127 in DMEM/F12 for 45 minutes at $37^{\circ} \mathrm{C}$, and then bathed with fresh DMEM/F12 
media on a rocker for 30 minutes at $37^{\circ} \mathrm{C}$ to allow complete dye de-esterification. Calcium transients were imaged in Tyrode solution supplemented with $10 \mu \mathrm{M}$ blebbistatin (Stemcell Technologies, 72402) to prevent motion artifacts. Cardiobundles were placed in a live imaging chamber heated at $37^{\circ} \mathrm{C}$, stimulated at $2-5 \mathrm{~Hz}$ using a field electrode, and fluorescence intensity videos were acquired using a fast EMCCD camera (iXonEM, Andor) affixed to a Nikon microscope. Changes in GCaMP6 or Fluo-4 fluorescence intensity relative to baseline fluorescence (df/f) calculated by Andor Solis software were used to measure calcium-transient amplitude.

For contractile force measurements, NRVM cardiobundles were moved to a custom force measurement setup to perform isometric force tests, as previously described $(54,56,90)$. Twitch responses to electrical stimulation at $2-5 \mathrm{~Hz}$ were recorded and analyzed using a custom MATLAB program. Twitch duration was calculated as the time between the $10 \%$ contraction and $90 \%$ relaxation phase (90). Force amplitude served as a measure of contractile strength of the cardiobundles.

ELISA. Twelve-week-old mice underwent LAD ligation and were injected with PBS or CSB. The first injection was i.m. into the myocardium immediately after injury. Additional i.v. injections were given at 2 , 4, and 6 days after MI. Hearts were isolated at 1, 4, or 8 days after MI and flash-frozen in liquid nitrogen. For native protein extraction, proteins were extracted from the isolated hearts using a mortar and pestle and rotor-stator homogenizer, with a native lysis buffer containing $0.5 \%$ Triton $\mathrm{X}-100$, protease inhibitors, and phosphatase inhibitors in PBS. The samples were centrifuged and proteins were collected for the ELISA. The levels of 16 cytokines were measured using 16-plex ELISA (110949MS, Quansys Biosciences) according to the manufacturer's instructions.

FACS. Twelve-week-old mice underwent LAD ligation and were injected with PBS or CSB, first by i.m. immediately after injury and then i.v. 2 days after MI. Single-cell suspensions from hearts harvested 4 days after $\mathrm{MI}$ were generated immediately before analysis by flow cytometry as previously described (91). Briefly, hearts were minced and digested in $2 \mathrm{mg} / \mathrm{mL}$ collagenase type 2 and $1.2 \mathrm{U} / \mathrm{mL}$ Dispase solution in PBS with $1 \mathrm{mM} \mathrm{CaCl}$ for 3 rounds of 15 minutes at $37^{\circ} \mathrm{C}$, followed by mechanical separation by pipetting. Digested samples were passed through a $40-\mu \mathrm{m}$ filter, washed, and suspended in FACS buffer (PBS with 0.5\% BSA and 2 mM EDTA) for staining. Samples were stained with antibodies from Milteny Biotec: CD45-Viogreen (catalog 130-110-638), CD11b-PE (catalog 130-109-285), Ly6G-Vioblue (catalog 130-110-449), F4/80-APC (catalog 130-102-379), and CCR2-APC-Vio770 (catalog 130-185-725). 7-AAD was used as a viability marker. Sample analysis was performed by standard procedure on a BD LSR II FACS machine.

Mac2 quantification. Mac2 area was quantified by pixel counting using FIJI software and calculated as percentage from the whole section area. For each heart, Mac2 area was quantified in 5 sequential sections located between the ligation site and the apex.

$B M D M$ culture. Bone marrow cells were isolated from the femur and tibia of 8-week-old C57BL/ 6JOlaHsd females. The cells were plated in macrophage differentiation medium (RPMI 1640, 10\% FBS, 1\% L-glutamine, 1\% penicillin/streptomycin, and $10 \mathrm{ng} / \mathrm{mL}$ M-CSF [PeproTech, 315-02]). Fifty percent of the medium was replaced with fresh medium every other day. After 6 days of culture, the medium was aspirated and replaced with fresh macrophage differentiation medium with the addition of $10 \mathrm{ng} / \mathrm{mL} \mathrm{Sal-}$ monella lipopolysaccharide (LPS) (Sigma-Aldrich). After 24 hours the LPS-containing medium was aspirated, cells were washed twice with PBS, and were treated with $50 \mu \mathrm{M}$ CSB in macrophage differentiation medium. Twenty-four hours later the cells were washed and RNA was purified.

$R N A$ purification and $q R T-P C R$. For BMDMs, total RNA was purified using TRIzol (TRI Reagent, Sigma-Aldrich, T9424). cDNA was synthesized using the High-Capacity cDNA Reverse Transcription kit (Applied Biosystems) according to the manufacturer's protocol. cDNA was loaded on a 96-well qRT-PCR plate. The qRT-PCR reaction was performed using SYBR Green PCR Master Mix (Applied Biosystems) on a StepOnePlus Real-Time PCR system (Applied Biosystems). Values for specific genes were normalized to $H P R T$ as housekeeping control. Primers used were HPRT: forward 5'-AGCGTCGTGATTAGCGATGA-3' and reverse 5'-GCAAGTCTTTCAGTCCTGTCC-3'; IL1 $\beta$ : forward 5'-GCAACTGTTCCTGAACTCAACT-3' and reverse 5'-ATCTTTTGGGGTCCGTCAACT-3'; IL10: forward 5'-GCTCTTACTGACTGGCATGAG-3' and reverse 5'-CGCAGCTCTAGGAGCATGTG-3'; TNF $\alpha$ : forward 5'-CTGAACTTCGGGGTGATCGG-3' and reverse 5'-GGCTTGTCACTCGAATTTTGAGA-3'; IL6: forward 5'-TAGTCCTTCCTACCCCAATTTCC-3' and reverse 5'-TTGGTCCTTAGCCACTCCTTC-3'.

Compound concentration. In vitro: For the high-throughput screening, all compounds were dissolved in DMSO/DDW to a concentration of $10 \mu \mathrm{M}$. CSB was dissolved in DDW to $10 \mu \mathrm{M}$ for all in vitro cardiac cell culture experiments, 10 and $50 \mu \mathrm{M}$ for cardiobundle experiments, and $50 \mu \mathrm{M}$ for BMDM experiments. 
Although no adverse effects were observed following administration of $50 \mu \mathrm{M}$ CSB and although the effects that were induced by $50 \mu \mathrm{M}$ CSB were observed at a lower concentration (following $10 \mu \mathrm{M}$ CSB treatment), it should be noted that $50 \mu \mathrm{M}$ is considered a relatively high concentration for small-molecule treatment. SAR molecules were diluted to $10 \mu \mathrm{M}$ in DDW. KN62 was dissolved in DMSO and diluted to 1,5 , or $10 \mu \mathrm{M}$ in cell medium for in vitro cardiac cell culture experiments. In vivo: CSB was diluted in PBS. Injection by i.m.: $50 \mu \mathrm{L}$ of $100 \mu \mathrm{M}$ stock solution, which is $0.005 \mu$ moles $(5 \mu \mathrm{g})$. Injections by i.v.: $100 \mu \mathrm{L}$ of $100 \mu \mathrm{M}$ stock solution, which is $0.01 \mu$ moles $(10 \mu \mathrm{g})$. KN62 was diluted in PBS. Injection by i.m.: $50 \mu \mathrm{L}$ of $25 \mu \mathrm{M}$ stock solution, which is 1.25 nmoles (902.3 ng). Injection by i.v.: $200 \mu \mathrm{L}$ of $7.5 \mu \mathrm{M}$ stock solution, which is $1.5 \mathrm{nmoles}$ (1.08 $\mu \mathrm{g})$. For all experiments, CSB was ordered from Sigma-Aldrich as a powder (C8679).

Statistics. Data are presented as mean \pm SEM or mean \pm SD according to experiment design as indicated in the figure legends. Comparisons between 2 groups were performed using Student's $t$ test. Comparisons among multiple groups were performed using 1-way ANOVA and the relevant post hoc test and multiple-comparisons correction according to experiment design, as indicated in the figure legends. Statistical analyses were calculated using GraphPad Prism 6. $P$ values less than 0.05 were considered significant. All experiments and results were analyzed in a blind manner.

Study approval. All animal experiments were approved by the Institutional Animal Care and Use Committee of the Weizmann Institute of Science.

\section{Author contributions}

OY designed, performed, and analyzed most of the experiments and wrote the manuscript. KW established the project and performed some in vitro and in vivo experiments. EB helped to design and perform in vitro experiments and designed the summary figure. DK and DL performed in vivo surgeries. HL and NB designed and performed the cardiobundle experiments. $\mathrm{HB}$ and NK helped to design, perform, and analyze the high-throughput screen. TE, AG, DR, and KBU performed in vitro experiments. OB performed pathological examination. ET supervised the project and wrote the manuscript.

\section{Acknowledgments}

This work was supported by grants from the European Research Council (ERC StG 281289, CM turnover, and ERC AdG 788194, CardHeal), the Britain-Israel Research and Academic Exchange (BIRAX), the Israel Science Foundation, Foundation Leducq Transatlantic Network of Excellence grant to ET and NB, and NIH grant U01HL134764 to NB.

Address correspondence to: Eldad Tzahor, Department of Molecular Cell Biology, Weizmann Institute of Science, Rehovot, Israel. Phone: 972.8.934.3715; Email: eldad.tzahor@weizmann.ac.i1.

1. Roger VL. Epidemiology of heart failure. Circ Res. 2013;113(6):646-659.

2. Writing Group Members, et al. Heart disease and stroke statistics-2016 update: A report from the American Heart Association. Circulation. 2016;133(4):e38-360.

3. Tzahor E, Poss KD. Cardiac regeneration strategies: Staying young at heart. Science. 2017;356(6342):1035-1039.

4. Hashimoto H, Olson EN, Bassel-Duby R. Therapeutic approaches for cardiac regeneration and repair. Nat Rev Cardiol. 2018;15(10):585-600.

5. Al Attar N, et al. Long-term (1 year) functional and histological results of autologous skeletal muscle cells transplantation in rat. Cardiovasc Res. 2003;58(1):142-148.

6. Jackson KA, et al. Regeneration of ischemic cardiac muscle and vascular endothelium by adult stem cells. J Clin Invest. 2001;107(11):1395-1402.

7. Quevedo HC, et al. Allogeneic mesenchymal stem cells restore cardiac function in chronic ischemic cardiomyopathy via trilineage differentiating capacity. Proc Natl Acad Sci USA. 2009;106(33):14022-14027.

8. Qiao H, et al. Long-term improvement in postinfarct left ventricular global and regional contractile function is mediated by embryonic stem cell-derived cardiomyocytes. Circ Cardiovasc Imaging. 2011;4(1):33-41.

9. Shiba Y, et al. Allogeneic transplantation of iPS cell-derived cardiomyocytes regenerates primate hearts. Nature. 2016;538(7625):388-391.

10. Menasché P. Cell therapy trials for heart regeneration - lessons learned and future directions. Nat Rev Cardiol. 2018;15(11):659-671.

11. Porrello ER, et al. Transient regenerative potential of the neonatal mouse heart. Science. 2011;331(6020):1078-1080

12. Haubner BJ, et al. Complete cardiac regeneration in a mouse model of myocardial infarction. Aging (Albany NY). 2012;4(12):966-977.

13. Poss KD, Wilson LG, Keating MT. Heart regeneration in zebrafish. Science. 2002;298(5601):2188-2190.

14. Jopling C, Sleep E, Raya M, Martí M, Raya A, Izpisúa Belmonte JC. Zebrafish heart regeneration occurs by cardiomyocyte dedifferentiation and proliferation. Nature. 2010;464(7288):606-609. 
15. Oberpriller JO, Oberpriller JC. Response of the adult newt ventricle to injury. J Exp Zool. 1974;187(2):249-253.

16. Bettencourt-Dias M, Mittnacht S, Brockes JP. Heterogeneous proliferative potential in regenerative adult newt cardiomyocytes. $J$ Cell Sci. 2003;116(Pt 19):4001-4009.

17. Soonpaa MH, Kim KK, Pajak L, Franklin M, Field LJ. Cardiomyocyte DNA synthesis and binucleation during murine development. Am J Physiol. 1996;271(5 Pt 2):H2183-H2189.

18. Patterson M, et al. Frequency of mononuclear diploid cardiomyocytes underlies natural variation in heart regeneration. Nat Genet. 2017;49(9):1346-1353.

19. D'Uva G, et al. ERBB2 triggers mammalian heart regeneration by promoting cardiomyocyte dedifferentiation and proliferation. Nat Cell Biol. 2015;17(5):627-638.

20. Mahmoud AI, et al. Meis1 regulates postnatal cardiomyocyte cell cycle arrest. Nature. 2013;497(7448):249-253.

21. Porrello ER, et al. Regulation of neonatal and adult mammalian heart regeneration by the miR-15 family. Proc Natl Acad Sci USA. 2013;110(1):187-192.

22. Eulalio A, et al. Functional screening identifies miRNAs inducing cardiac regeneration. Nature. 2012;492(7429):376-381.

23. Bassat $\mathrm{E}$, et al. The extracellular matrix protein agrin promotes heart regeneration in mice. Nature. 2017;547(7662):179-184.

24. Engel FB, Hsieh PC, Lee RT, Keating MT. FGF1/p38 MAP kinase inhibitor therapy induces cardiomyocyte mitosis, reduces scarring, and rescues function after myocardial infarction. Proc Natl Acad Sci USA. 2006;103(42):15546-15551.

25. Kühn B, et al. Periostin induces proliferation of differentiated cardiomyocytes and promotes cardiac repair. Nat Med. 2007;13(8):962-969.

26. Bersell K, Arab S, Haring B, Kühn B. Neuregulin1/ErbB4 signaling induces cardiomyocyte proliferation and repair of heart injury. Cell. 2009;138(2):257-270.

27. Xin M, et al. Hippo pathway effector Yap promotes cardiac regeneration. Proc Natl Acad Sci USA. 2013;110(34):13839-13844.

28. Nakada Y, et al. Hypoxia induces heart regeneration in adult mice. Nature. 2017;541(7636):222-227.

29. Yahalom-Ronen Y, Rajchman D, Sarig R, Geiger B, Tzahor E. Reduced matrix rigidity promotes neonatal cardiomyocyte dedifferentiation, proliferation and clonal expansion. Elife. 2015;4:e07455.

30. Zebrowski DC, Becker R, Engel FB. Towards regenerating the mammalian heart: challenges in evaluating experimentally induced adult mammalian cardiomyocyte proliferation. Am J Physiol Heart Circ Physiol. 2016;310(9):H1045-H1054.

31. Alvarez R, et al. Cardiomyocyte cell cycle dynamics and proliferation revealed through cardiac-specific transgenesis of fluorescent ubiquitinated cell cycle indicator (FUCCI). J Mol Cell Cardiol. 2019;127:154-164.

32. Qian L, et al. In vivo reprogramming of murine cardiac fibroblasts into induced cardiomyocytes. Nature. 2012;485(7400):593-598.

33. Sattler S, Fairchild P, Watt FM, Rosenthal N, Harding SE. The adaptive immune response to cardiac injury-the true roadblock to effective regenerative therapies? NPJ Regen Med. 2017;2:19.

34. Zlatanova I, Pinto C, Silvestre JS. Immune modulation of cardiac repair and regeneration: the art of mending broken hearts Front Cardiovasc Med. 2016;3:40.

35. Zhang R, et al. Calmodulin kinase II inhibition protects against structural heart disease. Nat Med. 2005;11(4):409-417.

36. Sossalla S, et al. Inhibition of elevated $\mathrm{Ca}^{2+} /$ calmodulin-dependent protein kinase II improves contractility in human failing myocardium. Circ Res. 2010;107(9):1150-1161.

37. Roseth S, Fykse EM, Fonnum F. Uptake of L-glutamate into rat brain synaptic vesicles: effect of inhibitors that bind specifically to the glutamate transporter. J Neurochem. 1995;65(1):96-103.

38. Bai F, et al. A novel allosteric inhibitor of macrophage migration inhibitory factor (MIF). J Biol Chem. 2012;287(36):30653-30663.

39. Normand A, Rivière E, Renodon-Cornière A. Identification and characterization of human Rad51 inhibitors by screening of an existing drug library. Biochem Pharmacol. 2014;91(3):293-300.

40. Nguyen T, et al. An ubiquitin-binding molecule can work as an inhibitor of ubiquitin processing enzymes and ubiquitin receptors. Biochem Biophys Res Commun. 2016;479(1):33-39.

41. Scholzen T, Gerdes J. The Ki-67 protein: from the known and the unknown. J Cell Physiol. 2000;182(3):311-322.

42. Baghbanzadeh A, Modirsaneie M, Emam G, Hajinezhad M. Microhandling of vesicular glutamate uptake modulate feeding in broilers. J Anim Physiol Anim Nutr (Berl). 2010;94(1):74-77.

43. Neale SA, Copeland CS, Salt TE. Effect of VGLUT inhibitors on glutamatergic synaptic transmission in the rodent hippocampus and prefrontal cortex. Neurochem Int. 2014;73:159-165.

44. Yu G, et al. The antinociceptive effects of intracerebroventricular administration of Chicago Sky Blue 6B, a vesicular glutamate transporter inhibitor. Behav Pharmacol. 2013;24(8):653-658.

45. Saxena T, et al. Nanocarrier-mediated inhibition of macrophage migration inhibitory factor attenuates secondary injury after spinal cord injury. ACS Nano. 2015;9(2):1492-1505

46. He Z, Yan L, Yong Z, Dong Z, Dong H, Gong Z. Chicago Sky Blue 6B, a vesicular glutamate transporters inhibitor, attenuates methamphetamine-induced hyperactivity and behavioral sensitization in mice. Behav Brain Res. 2013;239:172-176.

47. Pellicena P, Schulman H. CaMKII inhibitors: from research tools to therapeutic agents. Front Pharmacol. $2014 ; 5: 21$.

48. Anderson ME, Brown JH, Bers DM. CaMKII in myocardial hypertrophy and heart failure. J Mol Cell Cardiol. 2011;51(4):468-473.

49. Hund TJ, et al. Role of activated CaMKII in abnormal calcium homeostasis and $\mathrm{I}(\mathrm{Na})$ remodeling after myocardial infarction: insights from mathematical modeling. J Mol Cell Cardiol. 2008;45(3):420-428.

50. Willeford A, Suetomi T, Nickle A, Hoffman HM, Miyamoto S, Heller Brown J. CaMKII $\delta$-mediated inflammatory gene expression and inflammasome activation in cardiomyocytes initiate inflammation and induce fibrosis. JCI Insight. 2018;3(12):97054.

51. Beauverger $P$, et al. Reversion of cardiac dysfunction by a novel orally available calcium/calmodulin-dependent protein kinase II inhibitor, RA306, in a genetic model of dilated cardiomyopathy. Cardiovasc Res. 2019:cvz097.

52. Ai X, Curran JW, Shannon TR, Bers DM, Pogwizd SM. Ca2+/calmodulin-dependent protein kinase modulates cardiac ryanodine receptor phosphorylation and sarcoplasmic reticulum $\mathrm{Ca}^{2+}$ leak in heart failure. Circ Res. 2005;97(12):1314-1322.

53. Maier LS, Zhang T, Chen L, DeSantiago J, Brown JH, Bers DM. Transgenic CaMKIIdeltaC overexpression uniquely alters cardiac myocyte $\mathrm{Ca}^{2+}$ handling: reduced SR Ca ${ }^{2+}$ load and activated SR Ca ${ }^{2+}$ release. Circ Res. 2003;92(8):904-911.

54. Jackman CP, Carlson AL, Bursac N. Dynamic culture yields engineered myocardium with near-adult functional output. Biomaterials. 2016;111:66-79. 
55. Chen TW, et al. Ultrasensitive fluorescent proteins for imaging neuronal activity. Nature. 2013;499(7458):295-300.

56. Madden L, Juhas M, Kraus WE, Truskey GA, Bursac N. Bioengineered human myobundles mimic clinical responses of skeletal muscle to drugs. Elife. 2015;4:e04885.

57. Shadrin IY, et al. Cardiopatch platform enables maturation and scale-up of human pluripotent stem cell-derived engineered heart tissues. Nat Commun. 2017;8(1):1825.

58. Phillips RJ, Lutz M, Premack B. Differential signaling mechanisms regulate expression of CC chemokine receptor-2 during monocyte maturation. J Inflamm (Lond). 2005;2:14.

59. Sharma UC, et al. Galectin-3 marks activated macrophages in failure-prone hypertrophied hearts and contributes to cardiac dysfunction. Circulation. 2004;110(19):3121-3128.

60. Schuettauf F, et al. Administration of novel dyes for intraocular surgery: an in vivo toxicity animal study. Invest Ophthalmol Vis Sci. 2006;47(8):3573-3578.

61. Tsopelas C, Sutton R. Why certain dyes are useful for localizing the sentinel lymph node. J Nucl Med. 2002;43(10):1377-1382

62. Sueyoshi N, et al. Functional processing of nuclear $\mathrm{Ca}^{2+} /$ calmodulin-dependent protein kinase phosphatase (CaMKP-N): evidence for a critical role of proteolytic processing in the regulation of its catalytic activity, subcellular localization and substrate targeting in vivo. Arch Biochem Biophys. 2012;517(1):43-52.

63. Risse $\mathrm{E}$, et al. Identification of a compound that disrupts binding of amyloid- $\beta$ to the prion protein using a novel fluorescence-based assay. J Biol Chem. 2015;290(27):17020-17028.

64. Liguz-Lecznar M, Skangiel-Kramska J. Vesicular glutamate transporters (VGLUTs): the three musketeers of glutamatergic system. Acta Neurobiol Exp (Wars). 2007;67(3):207-218.

65. Zhang T, et al. The cardiac-specific nuclear delta(B) isoform of $\mathrm{Ca}^{2+} /$ calmodulin-dependent protein kinase II induces hypertrophy and dilated cardiomyopathy associated with increased protein phosphatase 2A activity. J Biol Chem. 2002;277(2):1261-1267.

66. Backs J, et al. The delta isoform of CaM kinase II is required for pathological cardiac hypertrophy and remodeling after pressure overload. Proc Natl Acad Sci USA. 2009;106(7):2342-2347.

67. Vagnozzi RJ, et al. An acute immune response underlies the benefit of cardiac adult stem cell therapy. bioRxiv. https://doi. org/10.1101/506626. Published December 26, 2018. Accessed October 22, 2019.

68. Han C, et al. Acute inflammation stimulates a regenerative response in the neonatal mouse heart. Cell Res. 2015;25(10):1137-1151.

69. van Amerongen MJ, Harmsen MC, van Rooijen N, Petersen AH, van Luyn MJ. Macrophage depletion impairs wound healing and increases left ventricular remodeling after myocardial injury in mice. Am J Pathol. 2007;170(3):818-829.

70. King KR, et al. IRF3 and type I interferons fuel a fatal response to myocardial infarction. Nat Med. 2017;23(12):1481-1487.

71. Gao XM, et al. Deletion of macrophage migration inhibitory factor protects the heart from severe ischemia-reperfusion injury: a predominant role of anti-inflammation. J Mol Cell Cardiol. 2011;50(6):991-999.

72. Kaikita K, Hayasaki T, Okuma T, Kuziel WA, Ogawa H, Takeya M. Targeted deletion of CC chemokine receptor 2 attenuates left ventricular remodeling after experimental myocardial infarction. Am J Pathol. 2004;165(2):439-447.

73. Gislason GH, et al. Risk of death or reinfarction associated with the use of selective cyclooxygenase-2 inhibitors and nonselective nonsteroidal antiinflammatory drugs after acute myocardial infarction. Circulation. 2006;113(25):2906-2913.

74. Bozkurt B, et al. Pathophysiologically relevant concentrations of tumor necrosis factor-alpha promote progressive left ventricular dysfunction and remodeling in rats. Circulation. 1998;97(14):1382-1391.

75. Mann DL. Innate immunity and the failing heart: the cytokine hypothesis revisited. Circ Res. 2015;116(7):1254-1268.

76. Lavine KJ, et al. Distinct macrophage lineages contribute to disparate patterns of cardiac recovery and remodeling in the neonatal and adult heart. Proc Natl Acad Sci USA. 2016;113(10):E1414

77. Abbate A, et al. Increased myocardial apoptosis in patients with unfavorable left ventricular remodeling and early symptomatic post-infarction heart failure. J Am Coll Cardiol. 2003;41(5):753-760.

78. Tatsumi T, et al. Cytokine-induced nitric oxide production inhibits mitochondrial energy production and impairs contractile function in rat cardiac myocytes. J Am Coll Cardiol. 2000;35(5):1338-1346.

79. Van Tassell BW, et al. Interleukin-1 trap attenuates cardiac remodeling after experimental acute myocardial infarction in mice. $J$ Cardiovasc Pharmacol. 2010;55(2):117-122.

80. Singh MV, et al. $\mathrm{Ca}^{2+} /$ calmodulin-dependent kinase II triggers cell membrane injury by inducing complement factor B gene expression in the mouse heart. J Clin Invest. 2009;119(4):986-996.

81. Weinreuter M, et al. CaM kinase II mediates maladaptive post-infarct remodeling and pro-inflammatory chemoattractant signaling but not acute myocardial ischemia/reperfusion injury. EMBO Mol Med. 2014;6(10):1231-1245.

82. Reuter S, Soonpaa MH, Firulli AB, Chang AN, Field LJ. Recombinant neuregulin 1 does not activate cardiomyocyte DNA synthesis in normal or infarcted adult mice. PLoS One. 2014;9(12):e115871.

83. Iismaa SE, et al. Cardiac hypertrophy limits infarct expansion after myocardial infarction in mice. Sci Rep. 2018;8(1):6114

84. Quaife-Ryan GA, et al. Multicellular transcriptional analysis of mammalian heart regeneration. Circulation. 2017;136(12):1123-1139.

85. Neef S, et al. Improvement of cardiomyocyte function by a novel pyrimidine-based CaMKII-inhibitor. J Mol Cell Cardiol. 2018;115:73-81.

86. Baylis RA, Gomez D, Mallat Z, Pasterkamp G, Owens GK. The CANTOS Trial: one important step for clinical cardiology but a giant leap for vascular biology. Arterioscler Thromb Vasc Biol. 2017;37(11):e174-e177.

87. Nahrendorf M. Myeloid cell contributions to cardiovascular health and disease. Nat Med. 2018;24(6):711-720.

88. Agah R, Frenkel PA, French BA, Michael LH, Overbeek PA, Schneider MD. Gene recombination in postmitotic cells. Targeted expression of Cre recombinase provokes cardiac-restricted, site-specific rearrangement in adult ventricular muscle in vivo. $J$ Clin Invest. 1997;100(1):169-179.

89. Madisen L, et al. A robust and high-throughput Cre reporting and characterization system for the whole mouse brain. Nat Neurosci. 2010;13(1):133-140.

90. Jackman C, Li H, Bursac N. Long-term contractile activity and thyroid hormone supplementation produce engineered rat myocardium with adult-like structure and function. Acta Biomater. 2018;78:98-110.

91. Pinto AR, et al. Revisiting cardiac cellular composition. Circ Res. 2016;118(3):400-409. 